\title{
Systematic Review
}

\section{High Precision Use of Botulinum Toxin Type A (BONT-A) in Aesthetics Based on Muscle Atrophy, Is Muscular Architecture Reprogramming a Possibility? A Systematic Review of Literature on Muscle Atrophy after BoNT-A Injections}

\author{
Alexander D. Nassif ${ }^{1, * \mathbb{D}}$, Ricardo F. Boggio ${ }^{2}$, Sheila Espicalsky ${ }^{3}$ and Gladstone E. L. Faria ${ }^{2}$ (D) \\ 1 Departamento de Pesquisa, Núcleo Nassif-Ensino Médico e Pesquisa, Belo Horizonte 30411-148, Brazil \\ 2 Departamento de Pesquisa, Instituto Boggio-Medicina Ensino e Pesquisa, Sao Paulo 04004-030, Brazil; \\ ricardoboggio@ricardoboggio.com.br (R.F.B.); gladstonefaria@hotmail.com (G.E.L.F.) \\ 3 Departamento de Pesquisa, Clínica Sheila Espicalsky, Vila Velha 29101-104, Brazil; \\ drasheila@sheilaespicalsky.com.br \\ * Correspondence: contato@alexandernassif.com.br
}

check for

updates

Citation: Nassif, A.D.; Boggio, R.F.;

Espicalsky, S.; Faria, G.E.L. High

Precision Use of Botulinum Toxin

Type A (BONT-A) in Aesthetics Based

on Muscle Atrophy, Is Muscular

Architecture Reprogramming a

Possibility? A Systematic Review of

Literature on Muscle Atrophy after

BoNT-A Injections. Toxins 2022, 14, 81.

https://doi.org/10.3390/

toxins14020081

Received: 3 December 2021

Accepted: 18 January 2022

Published: 21 January 2022

Publisher's Note: MDPI stays neutral with regard to jurisdictional claims in published maps and institutional affiliations.

Copyright: (C) 2022 by the authors. Licensee MDPI, Basel, Switzerland. This article is an open access article distributed under the terms and conditions of the Creative Commons Attribution (CC BY) license (https:// creativecommons.org/licenses/by/ $4.0 /)$.

\begin{abstract}
Improvements in Botulinum toxin type-A (BoNT-A) aesthetic treatments have been jeopardized by the simplistic statement: "BoNT-A treats wrinkles". BoNT-A monotherapy relating to wrinkles is, at least, questionable. The BoNT-A mechanism of action is presynaptic cholinergic nerve terminals blockage, causing paralysis and subsequent muscle atrophy. Understanding the real BoNT-A mechanism of action clarifies misconceptions that impact the way scientific productions on the subject are designed, the way aesthetics treatments are proposed, and how limited the results are when the focus is only on wrinkle softening. We designed a systematic review on BoNT-A and muscle atrophy that could enlighten new approaches for aesthetics purposes. A systematic review, targeting articles investigating BoNT-A injection and its correlation to muscle atrophy in animals or humans, filtered 30 publications released before 15 May 2020 in accordance with the Preferred Reporting Items for Systematic Reviews and Meta-Analyses (PRISMA) guidelines. Histologic analysis and histochemistry showed muscle atrophy with fibrosis, necrosis, and an increase in the number of perimysial fat cells in animal and human models; this was also confirmed by imaging studies. A significant muscle balance reduction of $18 \%$ to $60 \%$ after single or seriated BoNT-A injections were observed in 9 out of 10 animal studies. Genetic alterations related to muscle atrophy were analyzed by five studies and showed how much impact a single BoNT-A injection can cause on a molecular basis. Seriated or single BoNT-A muscle injections can cause real muscle atrophy on a short or long-term basis, in animal models and in humans. Theoretically, muscular architecture reprogramming is a possible new approach in aesthetics.
\end{abstract}

Keywords: botulinum toxins; type A; botox; muscular atrophy; muscle atrophy; wrinkles; ficial lines; aesthelics; esthetics; muscular architecture reprogramming

Key Contribution: A systematic review of literature on muscle atrophy after BoNT-A injections.

\section{Introduction}

Botulinum toxin type A (BoNT-A) has been historically used for the aesthetic treatment of facial lines. Although there are an increasing number of on-label uses to treat a variety of disorders using BoNT-A, when it comes to aesthetics, all the on-label approvals refer to facial lines [1]. Currently BoNT-A is approved by the FDA for the aesthetic treatment of forehead, glabellar, and lateral canthal lines, while in some other countries, such as Brazil, the on-label aesthetic approval is more generic and permits BoNT-A injections all over the face to treat facial lines [2,3]. The main point is that all the aesthetic on-label approvals 
concern facial lines only. Numerous published clinical trials objectify the improvement of facial lines after treatment with BoNT-A [4]. A multitude of articles aimed to compare the main brands of BoNT-A available on the market regarding the durability of the effect of softening wrinkles provided by these toxins [5]. Dose comparisons between BoNT-A brands generate misleading results because they are all different and are not interchangeable substances [6-8].

Despite differences in market brands, all currently marketed BoNT-A have one thing in common: a protein complex of $150 \mathrm{kDa}$ composed of a heavy chain (HC, $100 \mathrm{kDa})$ linked via a disulfide bond to a light chain (LC, $50 \mathrm{kDa}$ ) [9-11]. After a BoNT-A injection, the simplified mechanism of action cascade can be described based on its biochemical structure [12-17] (Figure 1).

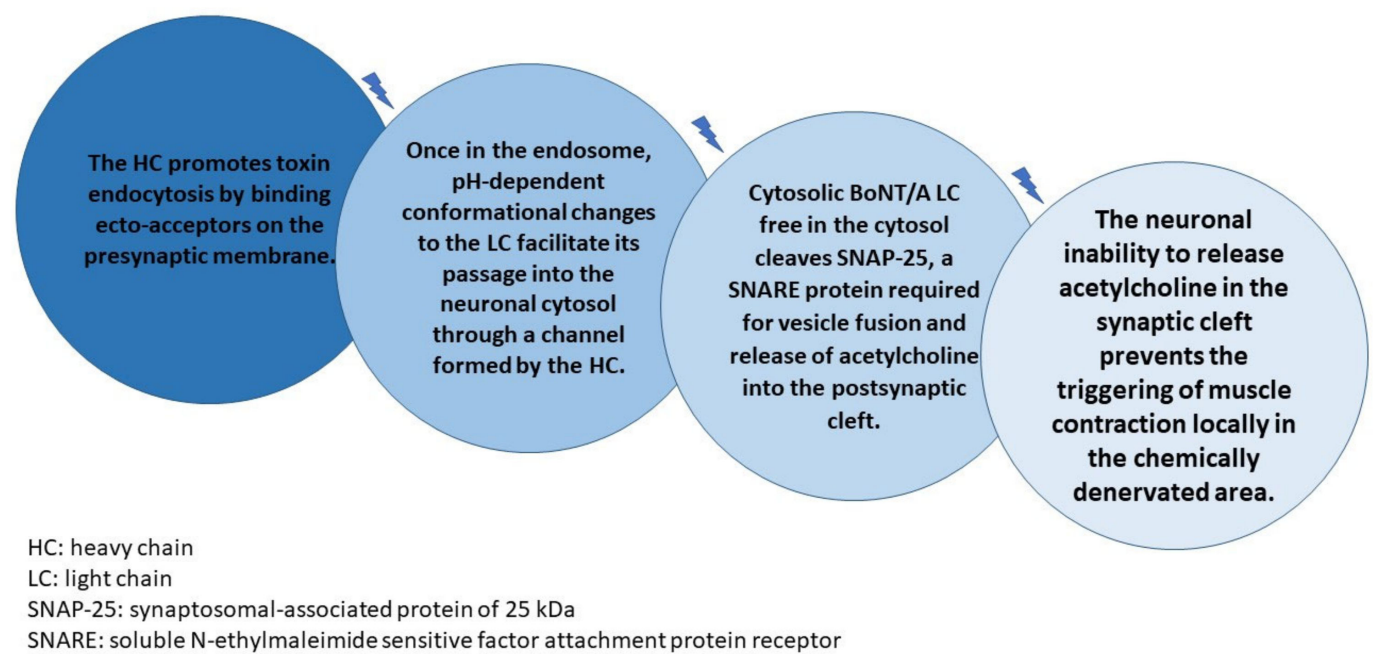

Figure 1. BoNT-A injection, the simplified mechanism of action cascade.

The whole cascade takes between 24 to $72 \mathrm{~h}$ to be completed after BoNT-A injection, and it is an irreversible process [18]. Once the SNAP-25 (synaptosomal-associated protein of $25 \mathrm{kDa}$ ) protein is inactivated, muscle contraction will only be reestablished after neuronal repair that depends on nerve sprouting and/or motor plate regeneration [19]. Although scientific evidence on this statement dates back to the 1970s [20], many still argue today about BoNT-A "durability" in relation to wrinkle control rather than studying the level of tissue damage caused by a BoNT-A injection and the time required for neuronal healing, as concerns aesthetics. The previous sentence is fundamental for the purpose of the new aesthetic approach of BoNT-A use in aesthetics that we intend to propose based on the real BoNT-A mechanism of action.

Many studies have demonstrated nerve terminal and nodal sprouting in the paralyzed nerves as early as two days after botulinum toxin injection [21,22]. Broadening the scope, studies on botulism have already provided a substrate to support the idea that the botulinum toxins durability for practical purposes is approximately 24 to $72 \mathrm{~h}$ and that the actual long-term effect of muscle paralysis depends only on nerve and muscle tissue regeneration processes. Treatment with antitoxin for patients with botulism, in order to be effective, should be started within 24 to $48 \mathrm{~h}$ of contamination, otherwise the already established neuronal chemical tissue injury is no longer reversible [23]. Once the disease is established by neuronal inability to release acetylcholine in the synaptic cleft of the neuromuscular junction, life support becomes essential, which is normally restricted to clinical care, with special attention to maintaining respiratory capacity, which requires mechanical ventilation for 2 to 6 months, until neuronal and muscular healing processes take place, restoring diaphragmatic and intercostal muscle function $[24,25]$.

Studies addressing counter-terrorism measures suggest the use of antidotes against BoNT-A in the event of a mass attack using BoNT-A as a chemical weapon. Only $1 \mathrm{~g}$ 
of BoNT-A in natura is capable of decimating 1 million humans, showing that it is a powerful and lethal toxin. All of the antidotes tested, even those capable of neuronal internalization, require concern regarding the therapeutic window, which must precede a chemical neuromuscular junction denervation of 24 to $72 \mathrm{~h}[24,26]$.

Understanding BoNT-A's real mechanism of action makes it possible to identify some semantic misconceptions that have been repeated historically since its first use for aesthetic purposes and that directly impact the way scientific productions on the subject are designed, the way aesthetics treatments are proposed, and how limited the results are when the focus is only on wrinkles softening. Considering the statements above and the questions raised below (Table 1), we designed a systematic review on BoNT-A and muscle atrophy that could enlighten new approaches for aesthetics purposes.

Table 1. Questions that should be answered, based on the evidence, after reading this paper.

\begin{tabular}{lc}
\multicolumn{1}{c}{ Questions } & Answers \\
\hline $\begin{array}{l}\text { Does the muscular impairment for contraction caused by BoNT-A really } \\
\text { treats facial lines or causes muscle atrophy? }\end{array}$ & $?$ \\
\hline What is the relation of BoNT-A muscle injections and muscle atrophy in the & $?$ \\
long term? & $?$ \\
\hline Is it possible to modulate the level of muscle atrophy through time by using & $?$ \\
BoNT-A? & $?$ \\
\hline What if we used muscle atrophy caused by BoNT-A injections to optimize & $?$ \\
muscle architecture for facial aesthetic purposes? & \\
\hline What would it be like to reinterpret articles written in the last 30 years & \\
focused mainly on facial lines unveiling this concept of muscle atrophy? \\
How many less subjective opportunities would arise? How classic BoNT-A \\
injections techniques would be impacted?
\end{tabular}

\section{Aims}

To conduct a systematic review of the literature regarding BoNT-A treatments and muscle atrophy that could support new perspectives in facial aesthetics and to propose a new reading for the aesthetic use of BoNT-A, no longer focusing on simple control of wrinkles and facial lines, but as a drug capable of selectively reprogramming long-term muscle strength and tonus through muscle atrophy. We will discuss the proposition that muscle architecture could be altered by creating areas of real atrophy-hyporesponsive or even irresponsive to acetylcholine stimuli for muscle contraction. The restoration of neuronal and muscular function would be based exclusively on the healing processes of these tissues.

\section{Method}

The present systematic review, targeting articles that investigate BoNT-A injections and its correlation to muscle atrophy in animals or humans, was conducted in a stepwise process for studies published before 15 May 2020 and in accordance with the Preferred Reporting Items for Systematic Reviews and Meta-Analyses (PRISMA) guidelines [27]. The search strategy, the flow diagram of study selection, and the data extraction are detailed below, because the review was not registered. By the time our independent research group tried to register the review at PROSPERO in 2020, we had already started article extraction. After October 2019, PROSPERO only accepted earlier registration.

STEP 1-PubMed/MEDLINE and BVS (Biblioteca Virtual em Saúde) databases were explored using the following Medical Subject Headings (MeSH) entry terms: "Botulinum Toxin Type A" OR "Botulinum A Toxin" OR "Botulinum Neurotoxin A" OR "Botox" AND combined with the MeSH entry terms "Muscle Atrophy" OR “Muscular Atrophy" (Table 2). The overlapping studies were excluded in STEP 1. 
Table 2. PubMed/MEDLINE and BVS (Biblioteca Virtual em Saúde) databases Search strategies.

\begin{tabular}{|c|c|}
\hline \multicolumn{2}{|c|}{ Four Search Strategies Used, Initially: } \\
\hline \multicolumn{2}{|c|}{$\begin{array}{c}\text { Search 1-PubMed/MEDLINE- }((((\text { BOTULINUM TOXIN TYPE A) OR (BOTULINUM } \\
\text { A TOXIN)) OR (BOTULINUM NEUROTOXIN A)) OR (BOTOX)) AND (MUSCLE } \\
\text { ATROPHY). }\end{array}$} \\
\hline \multicolumn{2}{|c|}{$\begin{array}{c}\text { Search 2-PubMed/MEDLINE- }((((\text { BOTULINUM TOXIN TYPE A) OR (BOTULINUM } \\
\text { A TOXIN)) OR (BOTULINUM NEUROTOXIN A)) OR (BOTOX)) AND (MUSCULAR } \\
\text { ATROPHY). }\end{array}$} \\
\hline \multicolumn{2}{|c|}{$\begin{array}{l}\text { Search 3-BVS-tw:((tw:(botulinum toxin type a)) OR (tw:(botulinum a toxin)) OR } \\
\text { (tw:(botulinum neurotoxin a)) OR (tw:(botox)) AND (tw:(muscle atrophy))). }\end{array}$} \\
\hline \multicolumn{2}{|c|}{$\begin{array}{l}\text { Search 4-BVS-tw:((tw:(botulinum toxin type a)) OR (tw:(botulinum a toxin)) OR } \\
\text { (tw:(botulinum neurotoxin a)) OR (tw:(botox)) AND (tw:(muscular atrophy))). }\end{array}$} \\
\hline \multicolumn{2}{|c|}{$\begin{array}{l}\text { To encompass all possible missing studies that could not be retrieved from Searches 1-4 } \\
\text { the preferred MeSH term entries "Botulinum Toxin Type A" and "Muscular Atrophy" } \\
\text { were matched with all their alternative MeSH term entries listed below: }\end{array}$} \\
\hline Botulinum toxin type A & Muscular atrophy \\
\hline Clostridium Botulinum Toxin Type A & Atrophies, Muscular \\
\hline Botulinum Toxin Type A & Atrophy, Muscular \\
\hline Botulinum A Toxin & Muscular Atrophies \\
\hline Toxin, Botulinum A & Atrophy, Muscle \\
\hline Clostridium botulinum A Toxin & Atrophies, Muscle \\
\hline Botulinum Neurotoxin A & Muscle Atrophies \\
\hline Neurotoxin A, Botulinum & Muscle Atrophy \\
\hline Meditoxin & Neurogenic Muscular Atrophy \\
\hline Botox & Atrophies, Neurogenic Muscular \\
\hline Neuronox & Atrophy, Neurogenic Muscular \\
\hline Oculinum & Muscular Atrophies, Neurogenic \\
\hline Vistabex & Muscular Atrophy, Neurogenic \\
\hline OnabotulinumtoxinA & Neurogenic Muscular Atrophies \\
\hline Onabotulinumtoxin A & Neurotrophic Muscular Atrophy \\
\hline Vistabel & Atrophies, Neurotrophic Muscular \\
\hline & Atrophy, Neurotrophic Muscular \\
\hline & Muscular Atrophies, Neurotrophic \\
\hline & Muscular Atrophy, Neurotrophic \\
\hline & Neurotrophic Muscular Atrophies \\
\hline
\end{tabular}

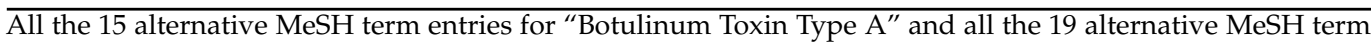
entries for "Muscle Atrophy" listed above were individually added to Search 1, Search 2, Search 3, and Search 4, one at a time, to check if any other study would be retrieved. No other search limits were added.

In STEP 2, the studies obtained in STEP 1 were screened by "title" and "abstract" by two independent researchers (A.D.N. and R.F.B.). Those not satisfying inclusion criteria or with exclusion criteria (Table 3) were excluded. The group of articles selected to proceed to the next step was determined through an interactive consensus process. Discrepancies were judged by a third reviewer (S.E.). 
Table 3. Inclusion and exclusion criteria.

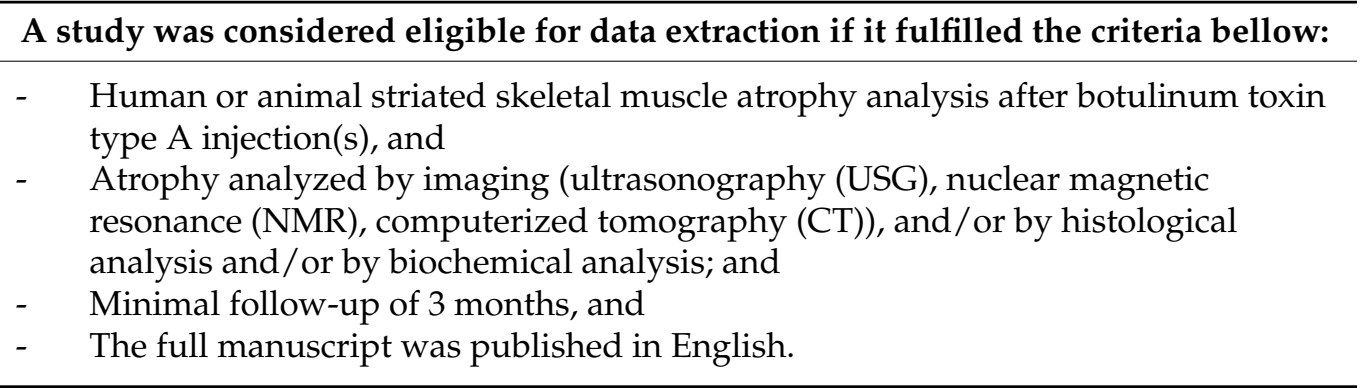

In STEP 3, the full text of all the potential articles selected in STEP 2 were obtained and carefully read to screen for those whose purposes were in accordance with the aim of the present review.

In STEP 4, the eligible studies in STEP 3 were thoroughly read, and data for each study were extracted and analyzed according to a PICO-like structured reading (Table 4).

Table 4. PICO-like structured reading of the eligible studies and data collection.

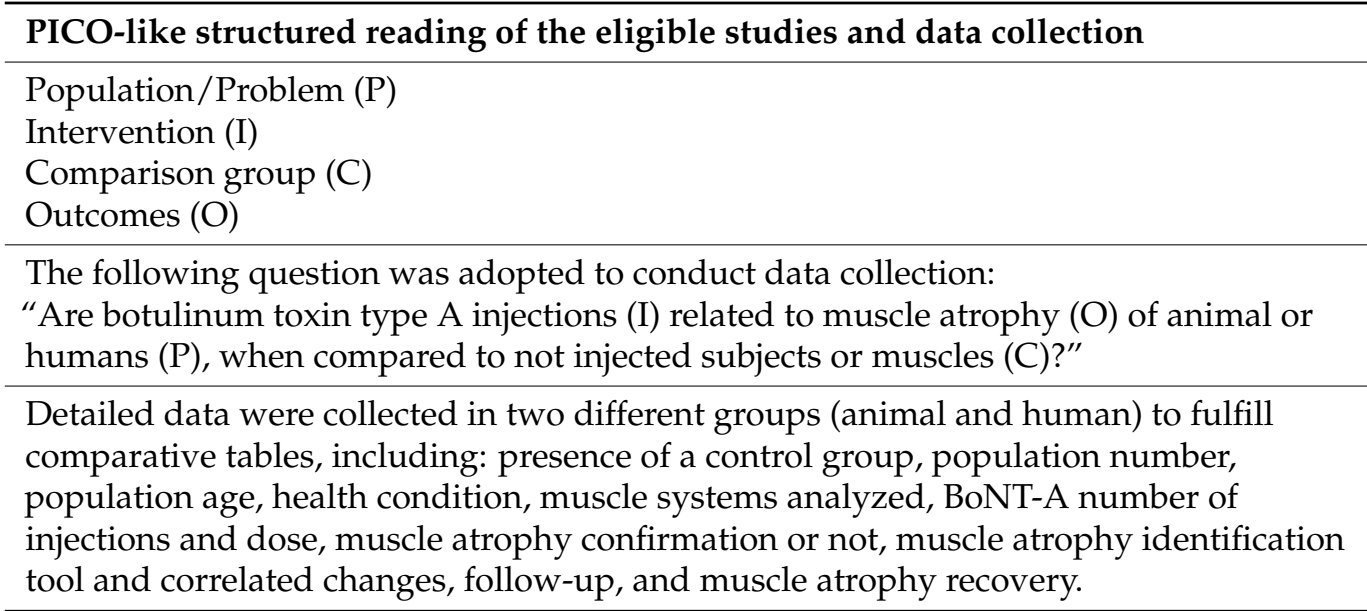

The methodological quality of the articles included in the study was evaluated using a specific scale developed based on STROBE (Strengthening the Reporting of Observational studies in Epidemiology) principles [28]. Each item was categorized, and the maximum global score was set to 26 (Table 5). 
Table 5. Quality analysis form used in the systematic review.

\section{Quality Analysis form Used in the Systematic Review.}

Q1 Is there in the abstract an explanation of what was done and found?

Q2 Is the scientific context clearly explained?

Q3 Are the objectives clearly stated?

Q4 Is the sampling size indicated?

Q5 If yes, is the sampling size statistically justified?

Q6 Are the characteristics of the subjects (height, weight, sex, healthy, or pathologic subject) described?

Q7 What is the design of the study? (0: retrospective study; 1 : case study; 2: prospective study).

Q8 Is there a control group? (0: no, 1: contralateral member or nonrandomized control group, 2: randomized control group).

Q9 How long is the follow up? (0: $\geq 3$ and $<6$ months; $1: \geq 6$ months and $<1$ year; $2: \geq 1$ year)

Q10 Is the reliability of the evaluation method clearly described?

Q11 Are the results interpretable?Q12 Are the limitations of the study discussed?Q13 Is the conclusion clearly stated?

0: no description; 1: limited description; 2: good description.

\section{Reults}

\subsection{Selection of the Studies}

From 191 articles initially identified after removing duplicates, thirty-five were deemed relevant after reading titles and abstracts. Thirty were included in the review (5 were excluded because they did not meet the selection criteria). Sixteen were animal studies and fourteen were human studies. The PRISMA Flow Diagram of Article Selection for Review is summarized in (Figure 2).
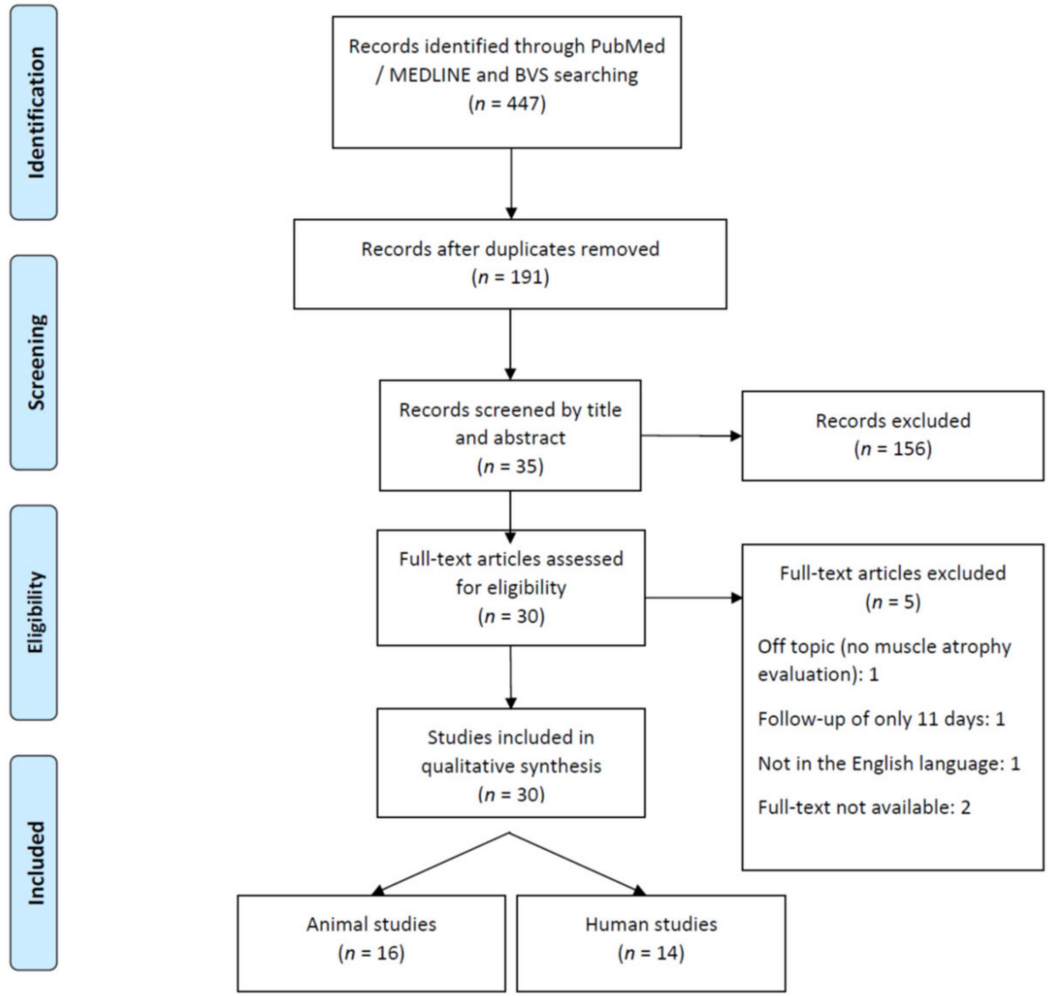

Figure 2. PRISMA-Flow Diagram of Article Selection for Review. 


\subsection{Quality of the Reviewed Articles}

The quality of the reviewed articles was highly variable and is summed up in Table 6 [29-58]. Most studies, 28/30, were prospective ones, with 13 well-controlled and randomized, but this subgroup was only of animal studies. The descriptive quality of the experimental protocol results, as well as their interpretations and conclusions, were adequate in most studies. The follow-up ranged from 3 months to 4 years.

Table 6. Quality assessment. ${ }^{* *}$ maximum global score $=26$.

\begin{tabular}{|c|c|c|c|c|c|c|c|c|c|c|c|c|c|c|}
\hline & Q1 & Q2 & Q3 & Q4 & Q5 & Q6 & Q7 & Q8 & Q9 & Q10 & Q11 & Q12 & Q13 & TOTAL ** \\
\hline Borodic (1992) [29] & 1 & 2 & 2 & 2 & 0 & 1 & 2 & 1 & 2 & 1 & 1 & 0 & 2 & 17 \\
\hline Hamjian (1994) [30] & 1 & 1 & 1 & 2 & 0 & 2 & 2 & 1 & 0 & 2 & 1 & 0 & 1 & 14 \\
\hline Ansved (1997) [31] & 2 & 2 & 2 & 2 & 0 & 2 & 1 & 1 & 2 & 1 & 1 & 0 & 1 & 17 \\
\hline Fanucci (2001) [32] & 2 & 2 & 2 & 2 & 0 & 2 & 2 & 1 & 0 & 2 & 2 & 0 & 2 & 19 \\
\hline To (2001) [33] & 2 & 2 & 2 & 2 & 0 & 2 & 2 & 1 & 2 & 1 & 2 & 0 & 2 & 20 \\
\hline Kim (2005) [34] & 2 & 2 & 1 & 2 & 0 & 2 & 2 & 0 & 2 & 1 & 2 & 0 & 2 & 18 \\
\hline Shen (2006) [35] & 2 & 2 & 2 & 2 & 0 & 2 & 2 & 2 & 1 & 2 & 2 & 0 & 2 & 21 \\
\hline Singer (2006) [36] & 2 & 2 & 2 & 2 & 0 & 2 & 2 & 0 & 1 & 1 & 2 & 2 & 2 & 20 \\
\hline Herzog (2007) [37] & 2 & 2 & 2 & 2 & 0 & 2 & 2 & 2 & 1 & 2 & 2 & 2 & 2 & 23 \\
\hline Frick (2007) [38] & 2 & 2 & 1 & 2 & 0 & 2 & 2 & 2 & 0 & 1 & 2 & 1 & 2 & 19 \\
\hline Kwon (2007) [39] & 2 & 2 & 2 & 2 & 0 & 2 & 2 & 2 & 1 & 2 & 2 & 1 & 2 & 22 \\
\hline Lee (2007) [40] & 2 & 2 & 2 & 2 & 0 & 2 & 2 & 0 & 2 & 1 & 2 & 0 & 2 & 19 \\
\hline Schroeder (2009) [41] & 2 & 2 & 2 & 2 & 0 & 1 & 2 & 1 & 2 & 1 & 2 & 0 & 2 & 19 \\
\hline Babuccu (2009) [42] & 2 & 2 & 2 & 2 & 0 & 2 & 2 & 2 & 0 & 2 & 2 & 0 & 2 & 20 \\
\hline Tsai (2010) [43] & 2 & 2 & 1 & 2 & 0 & 1 & 2 & 1 & 2 & 1 & 1 & 1 & 2 & 18 \\
\hline Fortuna (2011) [44] & 2 & 2 & 2 & 2 & 0 & 2 & 2 & 2 & 1 & 1 & 1 & 1 & 2 & 20 \\
\hline Fortuna (2013a) [45] & 2 & 2 & 2 & 2 & 0 & 1 & 2 & 2 & 1 & 1 & 2 & 1 & 2 & 20 \\
\hline Van Campenhout (2013) [46] & 2 & 2 & 2 & 2 & 0 & 1 & 2 & 0 & 1 & 2 & 2 & 2 & 2 & 20 \\
\hline Koerte (2013) [47] & 2 & 2 & 2 & 2 & 0 & 2 & 2 & 1 & 2 & 1 & 2 & 0 & 1 & 19 \\
\hline Fortuna (2013b) [48] & 2 & 2 & 2 & 2 & 0 & 2 & 2 & 2 & 2 & 1 & 2 & 2 & 1 & 22 \\
\hline Mukund (2014) [49] & 1 & 2 & 2 & 2 & 0 & 2 & 2 & 1 & 2 & 2 & 2 & 1 & 2 & 21 \\
\hline Fortuna (2015) [50] & 2 & 2 & 2 & 2 & 0 & 2 & 2 & 2 & 2 & 2 & 2 & 0 & 2 & 22 \\
\hline Caron (2015) [51] & 2 & 2 & 2 & 2 & 0 & 2 & 2 & 2 & 2 & 1 & 2 & 0 & 1 & 20 \\
\hline Valentine (2016) [52] & 2 & 2 & 2 & 2 & 0 & 2 & 2 & 1 & 2 & 1 & 2 & 1 & 1 & 20 \\
\hline Li (2016) [53] & 1 & 1 & 2 & 2 & 0 & 2 & 1 & 0 & 2 & 0 & 1 & 0 & 1 & 13 \\
\hline Kocaelli (2016) [54] & 2 & 2 & 2 & 2 & 0 & 2 & 2 & 2 & 0 & 2 & 2 & 1 & 2 & 21 \\
\hline Hart (2017) [55] & 2 & 2 & 2 & 2 & 0 & 2 & 2 & 2 & 2 & 2 & 1 & 2 & 1 & 22 \\
\hline Han (2018) [56] & 2 & 2 & 2 & 2 & 0 & 2 & 2 & 0 & 1 & 1 & 2 & 1 & 1 & 18 \\
\hline Alexander (2018) [57] & 2 & 2 & 2 & 2 & 0 & 2 & 2 & 1 & 1 & 2 & 2 & 2 & 2 & 22 \\
\hline Lima (2018) [58] & 2 & 2 & 2 & 2 & 0 & 2 & 2 & 2 & 0 & 1 & 2 & 0 & 2 & 19 \\
\hline
\end{tabular}




\subsection{Literature Analysis}

A general overview of the population type of the 30 studies is summarized in Table 7. All Animal studies had good quality control groups. Human studies, on the other hand, lacked control groups or had poor quality control groups.

Most animal studies used mature healthy animals. Human studies, on the other hand, used very heterogeneous subjects in relation to age (varying from children to 91-year-old adults) and health status.

Overall, there were very few studies regarding the facial mimetic musculature in humans-only two: Borodic (1992) [29] and Koerte (2013) [47]. The facial masticatory musculature represented mainly by the masseter muscle were studied in three human studies: To (2001) [33], Kim (2005) [34], Lee (2007) [40]; and three animal studies: Kwon (2007) [39], Babuccu (2009) [42], Kocaelli (2016) [54].

Numerical heterogenic population samples (from 1 to 383 subjects) and qualitative heterogenic samples, more specifically in human studies (healthy and subjects with different muscle disorders), were observed.

There was also heterogenic BoNT-A dose, BoNT-A brand types used in the studies and follow-up period, summarized in Table 8.

The methodological variability among the small number of studies made it mandatory to conduct an extensive evaluation based on the identification of muscle atrophy after BoNT-A injections registered separately via different tools in animal or human studies. The general findings are summarized in Section 4.3.1. (Animal Studies) and Section 4.3.2. (Human Studies), below.

\subsubsection{Animal Studies}

Muscle Balance

Muscle balance was measured in 10 out of 16 animal studies to evaluate muscle atrophy. Significant muscle balance reduction after seriated BoNT-A injections and after one single BoNT-A injection were observed in 9 out of 10 studies. The reduction varied from $18 \%$, Fortuna (2013b) [48], to $60 \%$, Fortuna (2011) [44], and there was a BoNT-A dose dependency/interval of injection association identified by Herzog (2007) [37], Frick (2007) [38], Tsai (2010) [43], Fortuna (2011) [44], Fortuna (2013b) [48], and Caron (2015) [51]. The higher the dose, the higher the muscle balance reduction. Long intervals between injections permitted partial muscle balance recovery. Only Fortuna (2015) [50] found no muscle balance alterations after 6 months of injection (Table 9).

\section{Optical and Electron Microscopy}

Hystologic (optical and electron microscopy) analysis and histochemistry showed profound muscle structure changes in animal models, such as sarcomere distortion, decrease in myofibrillar diameters, and myofibrillolysis/myonecrosis-Babuccu (2009) [42], Tsai (2010) [43], Kocaelli (2016) [54]. Significant reduction of percentage of contractile materialFrick (2007) [38], Fortuna (2011) [44], Fortuna (2013a) [45], Fortuna (2013b) [48], Fortuna (2015) [50]. Replacement of contractile fibers with fat, fatty infiltration, and increased collagen fibers forming perimysium-Herzog (2007) [37], Fortuna (2011) [44], Kocaelli (2016) [54] (Table 10).

Imaging

Kwon (2007) [39] showed a computed tomography (CT) scan rabbit masseter muscle volume reduction of up to $18.41 \%( \pm 3.15)$ after 6 months of a BoNT-A injection. Magnetic resonance imaging (MRI) was used in monkeys by Han (2018) [56] and showed significant paraspinal muscles atrophy after BoNT-A injections (Table 11).

\section{Molecular Biology}

Direct and indirect muscle atrophy identification via molecular biology was studied and is detailed in Tables 12 and 13. 
Table 7. Systematic review-Summary table of the results (PART 1).

\begin{tabular}{|c|c|c|c|c|c|}
\hline Author (Year) & Human/Animal & Control Group & Age & Population (Number) & Health Condition \\
\hline Borodic (1992) [29] & Human & Yes & 56-91 years & 14 & Blepharospasm/Meige's disease \\
\hline Hamjian (1994) [30] & Human & Contralateral muscle & 25-49 years & 10 & Healthy \\
\hline Ansved (1997) [31] & Human & Yes & $32-54$ years & 22 & Cervical dystonia \\
\hline Fanucci (2001) [32] & Human & Contraleteral Muscle & $29-54$ years & 30 & $\begin{array}{l}\text { Piriformis muscle syndrome } \\
\text { (PMS) }\end{array}$ \\
\hline To (2001) [33] & Human & Yes & 16-32 years & 15 & Masseteric muscle hypertrophy \\
\hline Kim (2005) [34] & Human & No & Teenagers-40s & 383 & Masseteric muscle hypertrophy \\
\hline Shen (2006) [35] & Animal (Sprague-Dawley rats) & Yes & 1 month & 56 & Healthy \\
\hline Singer (2006) [36] & Human & No & $16-40$ years & 8 & $\begin{array}{c}\text { Chronic anterior knee pain and } \\
\text { related } \\
\text { disability }\end{array}$ \\
\hline Herzog (2007) [37] & $\begin{array}{c}\text { Animal (New Zealand white } \\
\text { rabbits) }\end{array}$ & Yes & 1 year & 25 & Healthy \\
\hline Frick (2007) [38] & Animal (Sprague-Dawley rats) & Contralateral muscle & Mature & 39 & Healthy \\
\hline Kwon (2007) [39] & Animal (New Zealand rabbits) & Yes & 4 weeks & 21 & Healthy \\
\hline Lee (2007) [40] & Human & No & 20-29 years & 10 & Healthy (square face) \\
\hline Schroeder (2009) [41] & Human & Contralateral muscle & $31-47$ years & 2 & Healthy \\
\hline Babuccu (2009) [42] & Animal (Wistar rats) & Yes & 15-day-old & 49 & Healthy \\
\hline Tsai (2010) [43] & $\mathrm{CD}^{\circledR}(\mathrm{SD}) \mathrm{IGS}$ rats & Contralateral muscle & Mature & 60 & Healthy \\
\hline Fortuna (2011) [44] & $\begin{array}{c}\text { Animal } \\
\text { (New Zealand White rabbits) }\end{array}$ & Yes & 1 year & 20 & Healthy \\
\hline Fortuna (2013a) [45] & $\begin{array}{c}\text { Animal } \\
\text { (New Zealand White rabbits) }\end{array}$ & Yes & Mature & 17 & Healthy \\
\hline
\end{tabular}


Table 7. Cont.

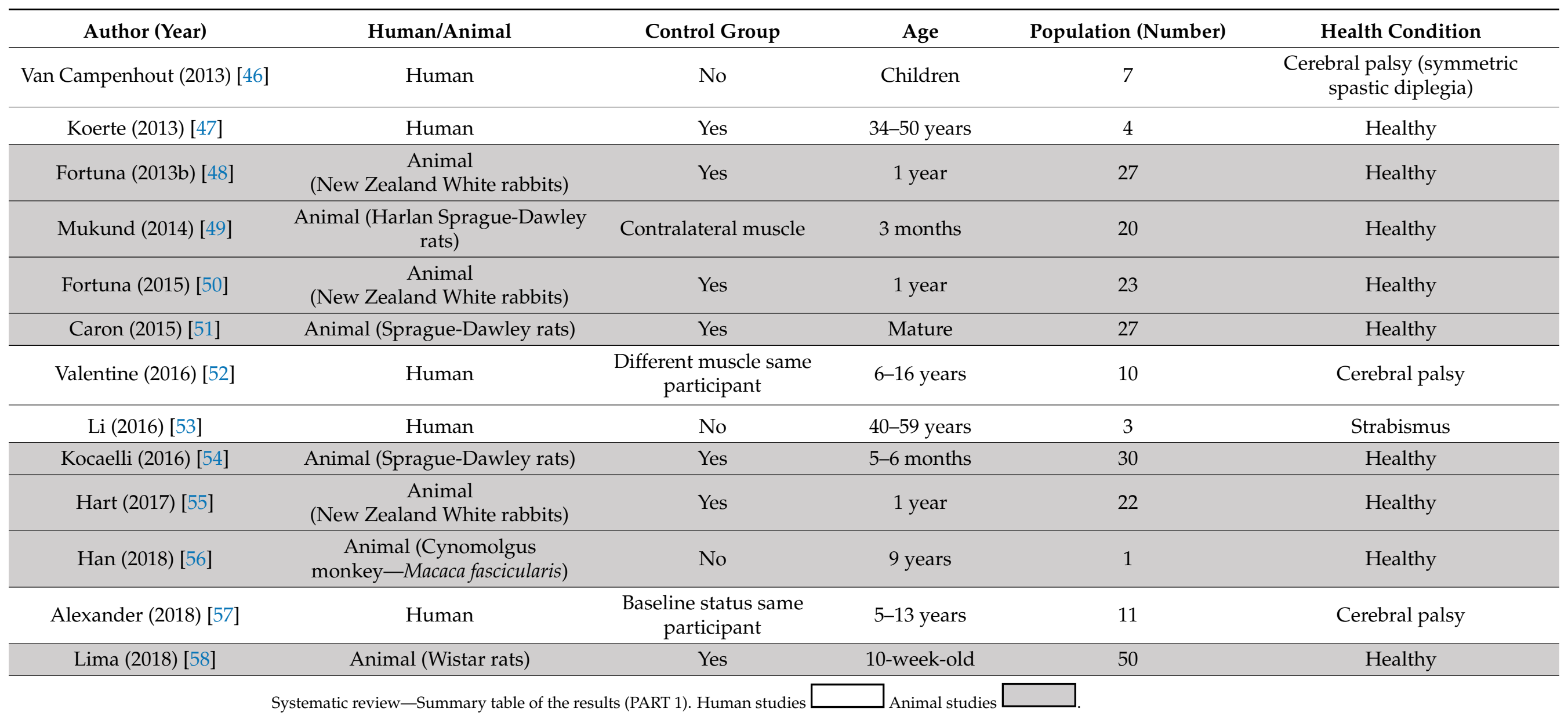


Table 8. Systematic review-Summary table of the results (PART 2).

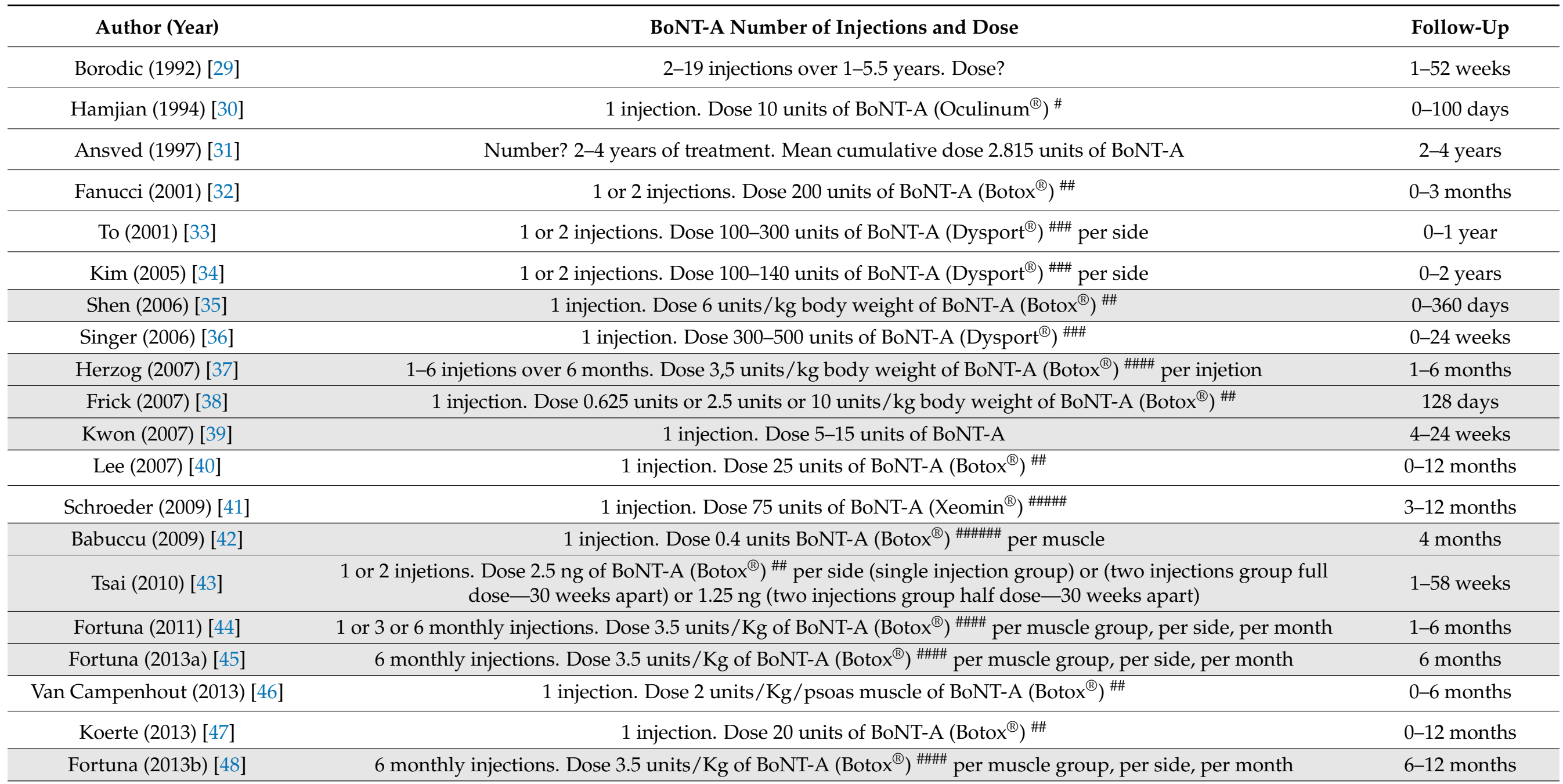


Table 8. Cont.

\begin{tabular}{|c|c|c|c|c|c|c|c|c|c|c|c|}
\hline \multicolumn{2}{|c|}{ Author (Year) } & \multicolumn{8}{|c|}{ BoNT-A Number of Injections and Dose } & \multicolumn{2}{|c|}{ Follow-Up } \\
\hline \multicolumn{2}{|c|}{ Mukund (2014) [49] } & \multicolumn{8}{|c|}{1 injection. Dose 6 units $/ \mathrm{Kg}$ of BoNT-A $\left(\text { Botox }^{\circledR}\right)^{\# \#}$ per side } & \multicolumn{2}{|c|}{ 1-52 weeks } \\
\hline \multicolumn{2}{|c|}{ Fortuna (2015) [50] } & \multicolumn{8}{|c|}{$\begin{array}{c}\left.\text { 1, 2, or } 3 \text { injections (every } 3 \text { months). Dose } 3.5 \text { units } / \mathrm{Kg} \text { of BoNT-A (Botox }{ }^{\circledR}\right)^{\# \# \# \#} \text { per muscle group, per side, per } \\
\text { injection }\end{array}$} & \multicolumn{2}{|c|}{ 6-12 months } \\
\hline \multicolumn{2}{|c|}{ Caron (2015) [51] } & \multicolumn{8}{|c|}{ 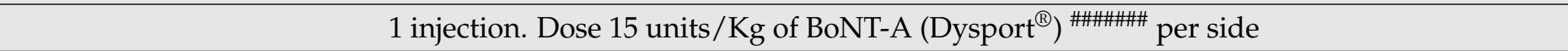 } & \multicolumn{2}{|c|}{ 12-400 days } \\
\hline \multicolumn{2}{|c|}{ Valentine (2016) [52] } & \multicolumn{8}{|c|}{ 1-15 injections. Dose $2-6$ units/Kg of BoNT-A $\left(\right.$ Botox $\left.^{\circledR}\right){ }^{\# \#}$ per side } & \multicolumn{2}{|c|}{3.5 months -3 years } \\
\hline \multicolumn{2}{|c|}{$\mathrm{Li}(2016)[53]$} & \multicolumn{8}{|c|}{ 1-2 injections. Dose 3.75-7.5 units of BoNT-A $\left(\right.$ Botox $\left.^{\circledR}\right){ }^{\# \#}$ per side } & \multicolumn{2}{|c|}{ 6-18 months } \\
\hline \multicolumn{2}{|c|}{ Kocaelli (2016) [54] } & \multicolumn{8}{|c|}{1 injection. Dose 0.5 units of BoNT-A $\left(\right.$ Botox $\left.^{\circledR}\right){ }^{\# \#}$ per muscle, per side } & \multicolumn{2}{|c|}{12 weeks } \\
\hline \multicolumn{2}{|c|}{ Hart (2017) [55] } & \multicolumn{8}{|c|}{ 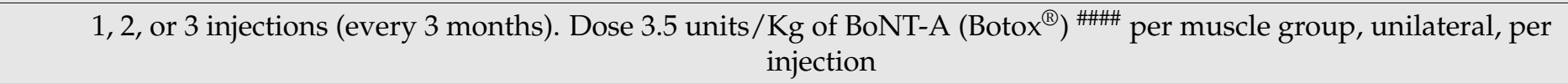 } & \multicolumn{2}{|c|}{$6-12$ months } \\
\hline \multicolumn{2}{|c|}{ Han (2018) [56] } & \multicolumn{8}{|c|}{ 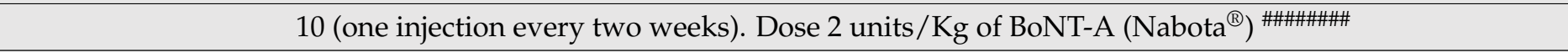 } & \multicolumn{2}{|c|}{ 0-21 weeks } \\
\hline \multicolumn{2}{|c|}{ Alexander (2018) [57] } & \multicolumn{8}{|c|}{1 injection. Dose $1.4-4.8$ units $/ \mathrm{Kg}$ of BoNT-A $\left(\right.$ Botox $\left.^{\circledR}\right){ }^{\# \#}$ per side } & \multicolumn{2}{|c|}{$0-25$ weeks } \\
\hline \multicolumn{2}{|c|}{ Lima (2018) [58] } & \multicolumn{8}{|c|}{1 injection. Dose 5 units of BoNT-A $\left(\right.$ Dysport $\left.^{\circledR}\right){ }^{\# \# \#}$ per side } & \multicolumn{2}{|c|}{12 weeks } \\
\hline & & \multicolumn{10}{|c|}{ 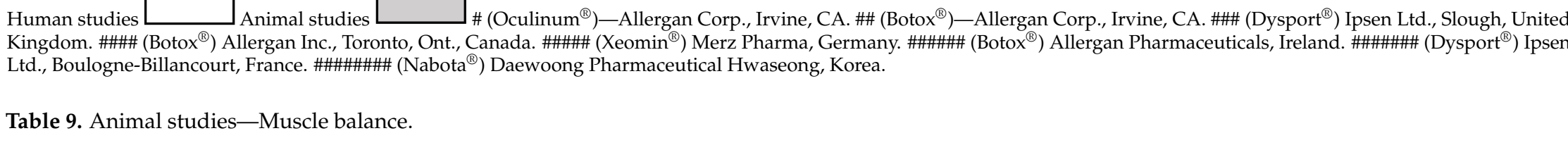 } \\
\hline $\begin{array}{l}\text { Muscle Atrophy } \\
\text { Identification Tool }\end{array}$ & $\begin{array}{l}\text { Herzog (2007) [37] } \\
\text { Quadriceps Femoris } \\
\text { 25 New Zealand White } \\
\text { Rabbits }\end{array}$ & $\begin{array}{c}\text { Frick (2007) [38] } \\
\text { Tibialis } \\
39 \text { Sprague-Dawley Rats }\end{array}$ & $\begin{array}{l}\text { Babuccu (2009) [42] } \\
\text { Masseter and Temporalis } \\
49 \text { Wistar Rats }\end{array}$ & $\begin{array}{l}\text { Tsai (2010) [43] } \\
\text { Gastrocnemius } \\
60 \text { CD }^{\mathbb{B}} \text { (SD) IGS Rats }\end{array}$ & $\begin{array}{c}\text { Fortuna (2011) [44] } \\
\text { Quadriceps Femoris } \\
\text { 20 New Zealand White } \\
\text { Rabbits }\end{array}$ & $\begin{array}{c}\text { Fortuna (2013a) [45] } \\
\text { Quadriceps Femoris } \\
\text { 20 New Zealand White } \\
\text { Rabbits }\end{array}$ & $\begin{array}{c}\text { Fortuna (2013b) [48] } \\
\text { Ouadriceps Femoris } \\
\text { 27 New Zealand White } \\
\text { Rabbits } \\
\end{array}$ & $\begin{array}{c}\text { Fortuna (2015) [50] } \\
\text { Quadriceps Femoris } \\
\text { 23 New Zealand White } \\
\text { Rabbits }\end{array}$ & $\begin{array}{r}\text { Caron } \\
\text { Gastro } \\
27 \text { Sprague } \\
\end{array}$ & $\begin{array}{l}15)[51] \\
\text { emius } \\
\text { awley Rats }\end{array}$ & $\begin{array}{l}\text { Lima (2018) [58] } \\
\text { Gastrocnemius } \\
50 \text { Wistar Rats }\end{array}$ \\
\hline & Wet muscle mass & Wet muscle mass & Wet muscle mass & Wet muscle mass & Wet muscle mass & Wet muscle mass & Wet muscle mass & Wet muscle mass & $\begin{array}{r}\text { Wet muscle n } \\
\text { weight/bod }\end{array}$ & $\begin{array}{l}\text { sand muscle } \\
\text { veight ratio }\end{array}$ & Wet muscle mass \\
\hline $\begin{array}{c}\text { Balance } \\
\text { (iimediately } \\
\text { post-sacritice muscle } \\
\text { harvestor muscle } \\
\text { harvest under general } \\
\text { anesthesia) }\end{array}$ & $\begin{array}{c}\text { Mean percent loss of } \\
\text { muscle mass of } 36 \% \text { at } \\
1 \text { month and } 49 \% \text { at } \\
6 \text { months. }\end{array}$ & $\begin{array}{l}\text { Significant (p< } 0.05 \text { ) decrease } \\
\text { of } 20 \% \text { in (group } 0.625 \text { units), } \\
33.4 \% \text { in (group } 2.5 \text { units) } \\
\text { and } 50 \% \text { in (group } 10 \text { units) } \\
\text { at day 128. No recovery at } \\
\quad \text { day } 128 .\end{array}$ & $\begin{array}{l}\text { Significicantly diminished } \\
p=0.0001 \text { (masseter and } \\
p=0.001 \text { (temporalis). } \\
\text { No recovery. }\end{array}$ & 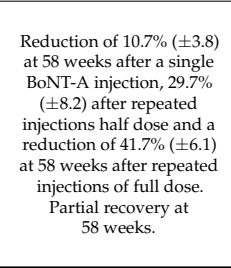 & $\begin{array}{l}\text { Significant atrophy } \\
\text { (p<0.0001). } \\
\text { Mean quadriceps femoris } \\
\text { muscle mass reduction of } \\
45 \% \text { ( m month group), 60\% } \\
\text { (3 months group), and } \\
56 \% \text { (6 months group). } \\
\text { No recovery. }\end{array}$ & $\begin{array}{l}\text { Significicant atrophy } \\
\text { (p<0.001). } \\
\text { Mean quadriceps femoris } \\
\text { muscle mass reduction of } \\
52 \% \text {. No recovery. }\end{array}$ & 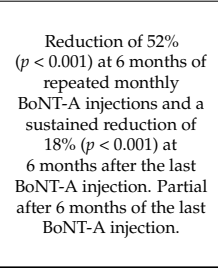 & $\begin{array}{l}\text { No alteration at } 6 \text { months } \\
\text { after the last BoNN-A } \\
\text { injection }(p>0.05) \text {. }\end{array}$ & 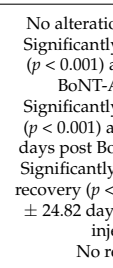 & 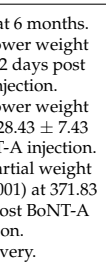 & $\begin{array}{c}\text { Significant reduction } \\
\text { of } 37 \%(p<0.001) \text {. }\end{array}$ \\
\hline
\end{tabular}


Table 10. Animal studies-Hystologic (optical and electron microscopy) analysis and histochemistry.

\begin{tabular}{|c|c|c|c|c|c|c|c|c|c|}
\hline $\begin{array}{l}\text { Muscle Atrophy } \\
\text { Identification Tool }\end{array}$ & $\begin{array}{c}\text { Herzog (2007) [37] } \\
\text { Quadriceps Femoris } \\
\text { 25 New Zealand White } \\
\text { Rabbits }\end{array}$ & $\begin{array}{c}\text { Frick (2007) [38] } \\
\text { Tibiailis } \\
\text { 39 Sprague-Dawley Rats }\end{array}$ & $\begin{array}{c}\text { Babuccu (2009) [42] } \\
\text { Masseter and Temporalis } \\
49 \text { Wistar Rats }\end{array}$ & $\begin{array}{c}\text { Tsai (2010) [43] } \\
\text { Gastrocnemius } \\
60 \mathrm{CD}^{\mathbb{8} \text { (SD) IGS Rats }}\end{array}$ & $\begin{array}{c}\text { Fortuna (2011) [44] } \\
\text { Quadriceps Femoris } \\
\text { 20 New Zealand White } \\
\text { Rabbits }\end{array}$ & $\begin{array}{c}\text { Fortuna (2013a) [45] } \\
\text { Quadriceps Femoris } \\
\text { 20 New Zealand White } \\
\text { Rabbits }\end{array}$ & $\begin{array}{c}\text { Fortuna (2013b) [48] } \\
\text { Quadriceps Femoris } \\
\text { 27 New Zealand White } \\
\text { Rabbits }\end{array}$ & $\begin{array}{c}\text { Fortuna (2015) [50] } \\
\text { Quadriceps Femoris } \\
\text { 23 New Zealand White } \\
\text { Rabbits }\end{array}$ & $\begin{array}{c}\text { Kocaelli (2016) [54] } \\
\text { Masseter and Gluteal } \\
\text { 30 Sprague-Dawley Rats }\end{array}$ \\
\hline \multirow{6}{*}{$\begin{array}{l}\text { Histologic analysis (optical } \\
\text { microscopy)/ } \\
\text { histochemistry }\end{array}$} & Muscle structure (qualitative) & Muscle structure (qualitative) & Muscle structure (qualitative) & Muscle structure (qualitative) & Muscle structure (qualitative) & Muscle structure (qualitative) & Muscle structure (qualitative) & Muscle structure (qualitative) & Muscle structure (qualitative \\
\hline & $\begin{array}{l}\text { Replacement of contractile } \\
\text { fibers with fat. }\end{array}$ & & & & $\begin{array}{l}\text { Fatty infiltration at } 3 \text { and } \\
6 \text { months (increased). } \\
\text { No recovery. }\end{array}$ & & & & $\begin{array}{l}\text { Increase in the collagen fiber } \\
\text { forming perinysium } \\
\text { around the striated muscle } \\
\text { cells at } 12 \text { weeks. }\end{array}$ \\
\hline & $\begin{array}{c}\text { Muscle structure (percentage } \\
\text { of contractile material) }\end{array}$ & $\begin{array}{c}\text { Muscle structure (percentage } \\
\text { of contractile material) }\end{array}$ & $\begin{array}{l}\text { Muscle structure (percentage } \\
\text { of contractile material) }\end{array}$ & $\begin{array}{l}\text { Muscle structure (percentage } \\
\text { of contractile material) }\end{array}$ & $\begin{array}{c}\text { Muscle structure (percentage } \\
\text { of contractile material) }\end{array}$ & $\begin{array}{c}\text { Muscle structure (percentage } \\
\text { of contractile material) }\end{array}$ & $\begin{array}{l}\text { Muscle structure (percentage } \\
\text { of contractile material) }\end{array}$ & $\begin{array}{l}\text { Muscle structure (percentage } \\
\text { of contractile material) }\end{array}$ & $\begin{array}{l}\text { Muscle structure (percentage } \\
\text { of contractile material) }\end{array}$ \\
\hline & & $\begin{array}{l}\text { Significant }(p<0.05) \text { decrease } \\
\text { at day } 128 \text {. No recovery at } \\
\text { day } 128 .\end{array}$ & & & 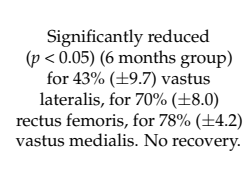 & $\begin{array}{c}\text { Reduction of } 36.1 \%( \pm 16.9), \\
(p<0.001) \text {. No recovery. }\end{array}$ & 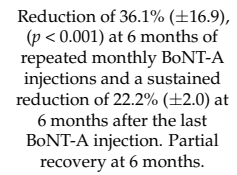 & 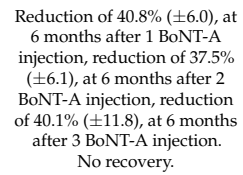 & \\
\hline & $\begin{array}{l}\text { Muscle structure (atrophy } \\
\text { scoring/quantitative } \\
\text { analysis) }\end{array}$ & $\begin{array}{l}\text { Muscle structure (atrophy } \\
\text { scoring/quantitative } \\
\text { analysis) }\end{array}$ & $\begin{array}{l}\text { Muscle structure (atrophy } \\
\text { scoring/quantitative } \\
\text { analysis) }\end{array}$ & $\begin{array}{l}\text { Muscle structure (atrophy } \\
\text { scoring/quantitative } \\
\text { analysis) }\end{array}$ & $\begin{array}{l}\text { Muscle structure (atrophy } \\
\text { scoring/quantititive } \\
\text { analysis) }\end{array}$ & $\begin{array}{l}\text { Muscle structure (atrophy } \\
\text { scoring/ quantititive } \\
\text { analysis }\end{array}$ & $\begin{array}{l}\text { Muscle structure (atrophy } \\
\text { scoring/quantititive } \\
\text { analysis }\end{array}$ & $\begin{array}{l}\text { Muscle structure (atrophy } \\
\text { scoring/quantitative } \\
\text { analysis) }\end{array}$ & $\begin{array}{l}\text { Muscle structure (atrophy } \\
\text { scoring/quantititaive } \\
\text { analysis) }\end{array}$ \\
\hline & & & $\begin{array}{l}\text { Stratification degree of the } \\
\text { muscle, nucleus } \\
\text { internalization, } \\
\text { multinucleation, myofibril } \\
\text { diameter, and myonecrosis } \\
\text { compatible with muscle } \\
\text { atrophy. No recovery at } \\
4 \text { months. }\end{array}$ & & & & & & $\begin{array}{l}\text { Significant }(p<0.001) \\
\text { decrease of diameters of } \\
\text { muscle fibers in bundles and } \\
\text { fascicles at } 12 \text { weeks. }\end{array}$ \\
\hline $\begin{array}{l}\text { Histologic analysis (electron } \\
\text { microscopy)/ } \\
\text { histochemistry }\end{array}$ & & & & $\begin{array}{l}\text { Sarcomere distorsion (mild } \\
\text { distruction at } 8 \text { weeks). } \\
\text { Partial reccovery at } 26 \text { weeks. }\end{array}$ & & & & & 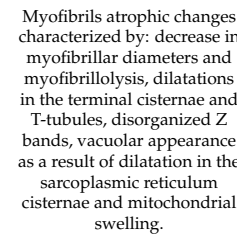 \\
\hline
\end{tabular}


Table 11. Animal studies-Imaging.

\section{Muscle Atrophy \\ Identification Tool}

Magnetic resonance
imaging (MRI)

imaging (MRI)

\section{Kwon (2007) [39] \\ Masseter \\ 21 New Zealand Rabbits}

\section{Han (2018) [56] \\ Paraspinal \\ 01 Cynomolgus Monkey-Macaca Fascicularis}

Muscle cross-sectional areas at T12-L1, L1-L2, L2-L3, L3-L4 and L4-L5 levels
Muscle cross-sectional areas at T12-L1, L1-L2, L2-L3, L3-L4, and L4-L5 levels

Significant atrophy with decreased cross-sectional areas by $4 \%, 2 \%, 8 \%, 12 \%$, and $8 \%$, respectively, at 21 weeks (the peak

was at 11 weeks). Partial recovery at 21 weeks.

\begin{tabular}{lc}
\hline & Muscle volume \\
\cline { 2 - 3 } Computed tomography & Muscle volume \\
Reduction of $19.72 \%( \pm 4.80)$ in Group 2 and \\
of $)$ scan $21.34 \%( \pm 5.37)$ in Group 3 at 8 weeks. \\
Reduction of $13.76 \%( \pm 5.34)$ in Group 2 and \\
of $18.41 \%( \pm 3.15)$ in Group 3 at 24 weeks. \\
Partial recovery at 24 weeks.
\end{tabular}

Table 12. Animal studies-Direct and indirect muscle atrophy identification via molecular biology.

\begin{tabular}{|c|c|}
\hline Molecular Biology Alterations & Articles \\
\hline $\begin{array}{l}\text { Upregulation of proapoptotic: anti-apoptotic protein ratio } \\
\text { ((Bax:Bcl-2)ratio) significantly had an } 83.3 \text { fold increase, peak at } \\
4 \text { weeks. } \\
p<0.01\end{array}$ & Tsai (2010) [43]. \\
\hline $\begin{array}{l}\text { Muscle substitution for adipose tissue determined by } \\
\text { adipocyte-related molecules upregulation of adiponectin (APN), } \\
\text { Leptin, adipocyte binding protein } 2 \text { (AP2), and adipogenic } \\
\text { lineage marker upregulation of peroxisome } \\
\text { proliferator-activated receptor } \gamma \text { (PPAR } \gamma \text { ). The APN, Leptin, } \\
\text { AP2, and PPAR } \gamma \text { were significantly upregulated after BoNT-A } \\
\text { injections. } \\
p<0.05\end{array}$ & Hart (2017) [55]. \\
\hline $\begin{array}{l}\text { Muscle atrophy inferred via molecular biology in regard to } \\
\text { upregulation of Transforming Growth Factor-beta TGF- } \beta \text {; } \\
\text { upregulation of Nuclear Factor-kappaB (NF-kB); upregulation } \\
\text { of p53/Cell cycle control; upregulation of Inhibitor of DNA } \\
\text { binding (ID) proteins-Id1, Id2, Id3, Id4, and muscle } \\
\text { RING-finger protein-1 (MuRF1) upregulation. }\end{array}$ & $\begin{array}{l}\text { Mukund (2014) [49]. } \\
\text { Fortuna (2015) [50]. }\end{array}$ \\
\hline $\begin{array}{l}\text { Muscle atrophy and muscle atrophy recovery response } \\
\text { indirectly identified via NMJ restoration (muscle-specific } \\
\text { receptor tyrosine kinase (MuSK) upregulation, nicotinic } \\
\text { acetylcholine receptor (nAChR) upregulation), protection } \\
\text { against muscle cell apoptosis (P21 protein upregulation), } \\
\text { myogenesis modulation/muscle regeneration (insulin-like } \\
\text { growth factor-1 (IGF-1) upregulation, myogenin upregulation, } \\
\text { and mitogen-activated protein kinase (MAPK) upregulation). }\end{array}$ & $\begin{array}{l}\text { Shen (2006) [35]. } \\
\text { Mukund (2014) [49]. } \\
\text { Fortuna (2015) [50]. }\end{array}$ \\
\hline
\end{tabular}


Table 13. Animal studies-Molecular biology.

\begin{tabular}{|c|c|c|c|c|c|}
\hline Muscle Atrophy Identification Tool & $\begin{array}{c}\text { Shen (2006) [35] } \\
\text { Gastrocnemus } \\
\text { 56 Sprague-Dawley Rats }\end{array}$ & $\begin{array}{c}\text { Tsai (2010) [43] } \\
\text { Gastrocnemius } \\
60 \mathrm{CD}^{\circledR} \text { (SD) IGS Rats }\end{array}$ & $\begin{array}{c}\text { Mukund (2014) [49] } \\
\text { Tibialis Anterior } \\
\text { 20 Sprague-Dawley Rats }\end{array}$ & $\begin{array}{c}\text { Fortuna (2015) [50] } \\
\text { Quadriceps Femoris } \\
\text { 23 New Zealand White Rabbits }\end{array}$ & $\begin{array}{c}\text { Hart (2017) [55] } \\
\text { Quadriceps Femoris } \\
\text { 22 New Zealand White Rabbits }\end{array}$ \\
\hline \multirow{16}{*}{$\begin{array}{l}\text { Molecular biology } \\
\text { (Real-Time Quantitative Polymerase Chain } \\
\text { Reaction (PCRR), and //or Microarray Data } \\
\text { Analysis, and/or Western blot analysis) }\end{array}$} & \multicolumn{5}{|c|}{ Indirect atrophy identification via upregulation of gene and molecule expression signaling neuromuscular junction (NM) restoration, protection against muscle cell apoptosis, myogenesis modulation/muscle regeneration. } \\
\hline & NMJ restoration & NMJ restoration & NMJ restoration & NMJ restoration & NMJ restoration \\
\hline & $\begin{array}{l}\text { Muscle-specific receptor tyrosine kinase (MuSK) } \\
\text { significant } 4 \text { upregulation }(p<0.05) \text { from day } 3 \text { to day } 60 \\
\text { Nicotinic acelylcholine receptor (nAChR) significant } \\
\text { upregulation }(p<0.05 \text { ) from day } 3 \text { to day } 14\end{array}$ & & & & \\
\hline & Protection against muscle cell apoptosis & Protection against muscle cell apoptosis & Protection against muscle cell apoptosis & Protection against muscle cell apoptosis & Protection against muscle cell apoptosis \\
\hline & $\begin{array}{l}\text { P21 protein significant }(p<0.05) \text { upregulation from } \\
\text { day } 3 \text { to day } 30\end{array}$ & & & & \\
\hline & Myogenesis modulation/muscle regeneration & Myogenesis modulation/muscle regeneration & Myogenesis modulation/muscle regeneration & Myogenesis modulation/muscle regeneration & Myogenesis modulation/muscle regeneration \\
\hline & $\begin{array}{l}\text { Insulin-like growth factor-1 (IGF-1) significant } \\
\text { urpegulation }(p<<0.05 \text { ) from day 3to day } 60 \\
\text { Myogenin significant upregulation }(p<0.05) \text { from day } 3 \\
\text { to day } 90\end{array}$ & & & & \\
\hline & Myogenesis modulation/muscle regeneration & Myogenesis modulation/muscle regeneration & Myogenesis modulation/muscle regeneration & Myogenesis modulation/muscle regeneration & Myogenesis modulation/muscle regeneration \\
\hline & & & & $\begin{array}{l}\text { Insulin-like growth factor-1 (IGF-1) significant } \\
\text { upregulation }(p<0.05 \text { ) (at } 6 \text { months) } \\
\text { Recovery not evaluated }\end{array}$ & \\
\hline & $\begin{array}{l}\text { Direct atrophy identification via upregulation of } \\
\text { proapoptotic: } \\
\text { anti-apoptotic protein ratio (Bax:Bcl-2) }\end{array}$ & $\begin{array}{l}\text { Direct atrophy identification via upregulation of } \\
\text { proapoptotic: } \\
\text { anti-apoptotic protein ratio (Bax:Bcl-2) }\end{array}$ & $\begin{array}{l}\text { Direct atrophy identification via upregulation of } \\
\text { proapoptotic: } \\
\text { anti-apoptotic protein ratio (Bax:Bcl-2) }\end{array}$ & $\begin{array}{l}\text { Direct atrophy identification via upregulation of } \\
\text { proapoptotic: } \\
\text { anti-apoptotic protein ratio (Bax:Bcl-2) }\end{array}$ & $\begin{array}{l}\text { Direct atrophy identification via upregulation of } \\
\text { proapoptotic: } \\
\text { anti-apopototic protein ratio (Bax:Bcl-2) }\end{array}$ \\
\hline & & $\begin{array}{l}\text { Ratio significantly } 83.3 \text { fold increase }(p<0.01) \text { (peak } \\
\text { at } 4 \text { weeks) } \\
\text { Recovery at } 8 \text { weeks }\end{array}$ & & & \\
\hline & & & & $\begin{array}{c}\text { TGF- } \beta \text { significantly upregulated }(p<0.05) \\
\text { (at } 6 \text { monthls) } \\
\text { Recovery not evaluated }\end{array}$ & \\
\hline & $\begin{array}{l}\text { Direct atrophy identification via muscle RING-finger } \\
\text { protein-1 (MuRF1) }\end{array}$ & $\begin{array}{l}\text { Direct atrophy identification via muscle RING-finger } \\
\text { protein-1 (MuRF1) }\end{array}$ & $\begin{array}{l}\text { Direct atrophy identification via muscle RING-finger } \\
\text { protein-1 (MuRF1) }\end{array}$ & $\begin{array}{l}\text { Direct atrophy identification via muscle RING-finger } \\
\text { protein-1 (MuRF1) }\end{array}$ & $\begin{array}{l}\text { Direct atrophy identification via muscle RING-finger } \\
\text { protein-1 (MuRF1) }\end{array}$ \\
\hline & & & & $\begin{array}{l}\text { MuRF1 significantly upregulated }(p<0.05) \\
\text { (at } 6 \text { months) } \\
\text { Recovery not evaluated }\end{array}$ & \\
\hline & $\begin{array}{l}\text { Direct atrophy identification via muscle substitution for } \\
\text { adipose tissue. } \\
\text { Adipocyte-related molecules upregulation of } \\
\text { adiponectin (APN), } \\
\text { Leptin, adipocyte binding protein 2(AP2), and } \\
\text { adipogenic lineage marker upregulation of peroxisome } \\
\text { proliferator-activated receptor } \gamma(\mathrm{PPAR} \gamma \text { ) }\end{array}$ & 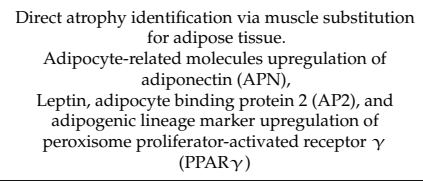 & 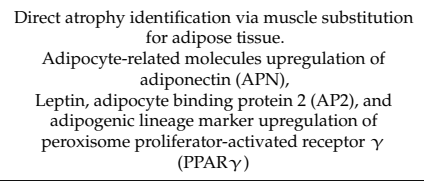 & 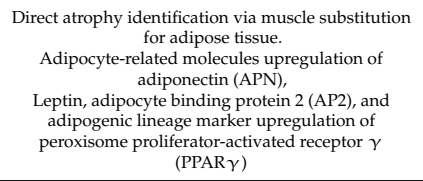 & 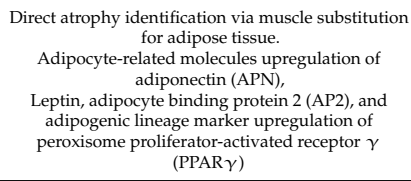 \\
\hline & & & & & $\begin{array}{l}\text { APN, Leptin, AP2, and PPAR } \gamma \text { significantly } \\
\text { upregulated ( } p<0.05 \text { ) (at } 6 \text { monthts after } 3 \text { BoNTT-A } \\
\text { injections every } 3 \text { month, except for Leptin, which } \\
\text { had partial recovery after } 3 \text { BoNT-A injections) }\end{array}$ \\
\hline
\end{tabular}




\subsubsection{Human Studies}

\section{Optical and Electron Microscopy}

Histologic (optical and electron microscopy) analysis and histochemistry showed results in humans similar to those found in animal models. Muscle atrophy (atrophic muscle fibers, myofibrillar disorganization, fibrosis, necrosis, and increase of the number of perimysial fat cells) were well-documented by Kim (2005) [34], Schroeder (2009) [41], Valentine (2016) [52], and Li (2016) [53]. The Orbicularis oculi muscle showed that the morphometric measurements of muscle fibers reduced, with an irregular diameter at 3 months after BoNT-A injections, $(p<0.05)$. Ansved (1997) [31] showed a mean diameter reduction of type IIB striated muscle fibers (Vastus lateralis) of $19.6 \%$ after $2-4$ years of BoNT-A treatement $(p<0.05)$. Partial recovery of the changes described above were seen in some articles (Table 14).

Table 14. Human studies-Histologic (optical and electron microscopy) analysis and histochemistry.

\begin{tabular}{|c|c|c|c|c|c|c|}
\hline $\begin{array}{l}\text { Muscle Atrophy } \\
\text { Identification Tool }\end{array}$ & $\begin{array}{l}\text { Borodic (1992) [29] } \\
\text { Orbicularis Oculi } \\
14\end{array}$ & $\begin{array}{c}\text { Ansved (1997) [31] } \\
\text { Vastus Lateralis } \\
\text { (Non-Target Muscle) } \\
22\end{array}$ & $\begin{array}{l}\text { Kim (2005) [34] } \\
\text { Masseter } \\
\quad 383\end{array}$ & $\begin{array}{l}\text { Schroeder (2009) [41] } \\
\text { Gastrocnemius } \\
2\end{array}$ & $\begin{array}{l}\text { Valentine (2016) [52] } \\
\text { Gastrocnemius } \\
10\end{array}$ & $\begin{array}{c}\text { Li (2016) [53] } \\
\text { Medial Rectus (Extraocular } \\
\text { Muscle) } \\
3\end{array}$ \\
\hline \multirow{4}{*}{$\begin{array}{l}\text { Histologic analysis (optical } \\
\text { microscopy)/ } / \\
\text { histochemistry }\end{array}$} & $\begin{array}{c}\text { Morphometric } \\
\text { measurements of muscle } \\
\text { fibers }\end{array}$ & $\begin{array}{c}\text { Morphometric } \\
\text { measurements of muscle } \\
\text { fibers }\end{array}$ & $\begin{array}{c}\text { Morphometric } \\
\text { measurements of muscle } \\
\text { fibers }\end{array}$ & $\begin{array}{c}\text { Morphometric } \\
\text { measurements of muscle } \\
\text { fibers }\end{array}$ & $\begin{array}{l}\text { Morphometric } \\
\text { measurements of muscle } \\
\text { fibers }\end{array}$ & $\begin{array}{l}\text { Morphometric measurements } \\
\text { of muscle fibers }\end{array}$ \\
\hline & $\begin{array}{c}\text { Reduced and irregular } \\
\text { diameter at } 3 \text { months } \\
(p<0.05) \text {. Partial recovery at } \\
6 \text { months. }\end{array}$ & $\begin{array}{l}\text { Mean diameter reduction of } \\
\text { type IIB fibers of } 19.6 \% \text { after } \\
2-4 \text { years of BoNT-A } \\
\text { treatement, }(p<0.05) .\end{array}$ & & & & \\
\hline & Muscle structure & Muscle structure & Muscle structure & Muscle structure & Muscle structure & Muscle structure \\
\hline & & & $\begin{array}{l}\text { Muscle atrophy, necrosis, } \\
\text { and hyaline } \\
\text { degeneration at } 4 \text { months. }\end{array}$ & $\begin{array}{l}\text { Muscle atrophy and Mild } \\
\text { increase of the } \\
\text { number of perimysial fat } \\
\text { cells. Muscle fiber area } \\
\text { reduction of } 24 \% \text { at } \\
12 \text { months. Partial recovery } \\
\text { at } 12 \text { months. }\end{array}$ & Muscle atrophy. & $\begin{array}{l}\text { Fibrosis with no identifiable } \\
\text { muscle fibers. }\end{array}$ \\
\hline \multirow[b]{2}{*}{$\begin{array}{l}\text { Histologic analysis (electron } \\
\text { microscopy)/ } \\
\text { histochemistry }\end{array}$} & Muscle ultrastructure & Muscle ultrastructure & Muscle ultrastructure & Muscle ultrastructure & Muscle ultrastructure & Muscle ultrastructure \\
\hline & & & & $\begin{array}{c}\text { Muscle atrophy of a } \\
\text { considerable } \\
\text { number of muscle fibers at } \\
12 \text { months. Partial recovery } \\
\text { at } 12 \text { months. }\end{array}$ & $\begin{array}{l}\text { Atrophic muscle fibers, } \\
\text { Myofibrillar } \\
\text { disorganization, } \\
\text { redundant basal lamina, } \\
\text { cores, and wrinkling of } \\
\text { the sarcolemmal } \\
\text { membrane. }\end{array}$ & \\
\hline
\end{tabular}

Imaging

All the 10 human studies that evaluated images to measure muscle atrophy after BoNT-A treatments showed signs of muscle atrophy, irrespective of the technology used: ultrasound, MRI, CT scan, or cephalometry. Muscle atrophy was registered in the short term (42 days to 3 months) and in the long term (up to 2 years). No full recovery was identified (Table 15). 
Table 15. Human studies-Imaging

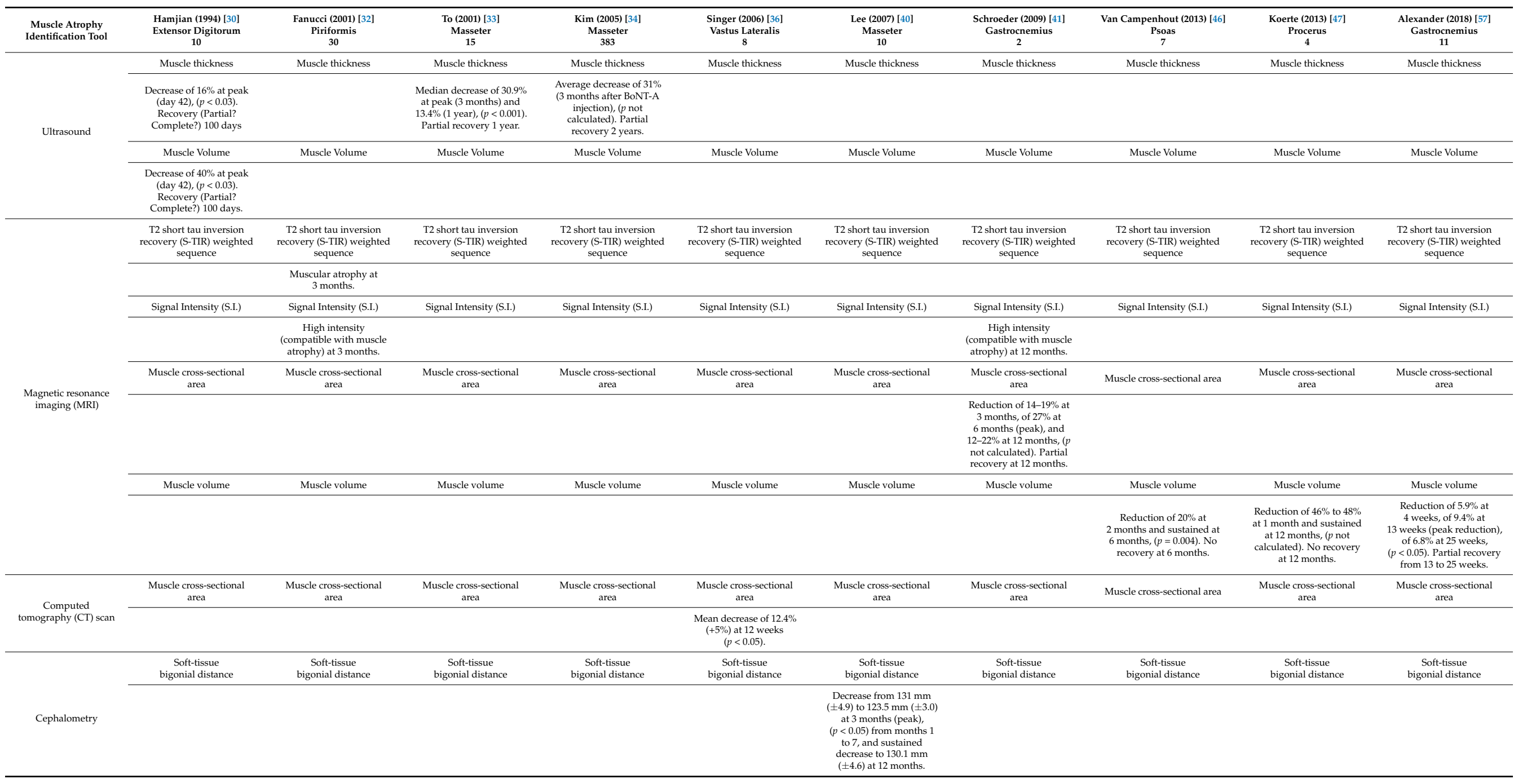




\section{Discussion}

The use of BoNT-A for cosmetic purposes is a fast-growing procedure, with more than six million treatments performed by plastic surgeons in the year 2018 alone [59]. Despite this significant number, we believe that improvements in BoNT-A aesthetic treatments have been jeopardized by the famous, but simplistic, statement used by the media, patients, and doctors: "BoNT-A treats wrinkles". BoNT-A monotherapy relating to wrinkles is, at least, questionable. The BoNT-A mechanism of action is presynaptic cholinergic nerve terminals blockage by inhibition of the release of acetylcholine, causing paralysis and subsequent functional denervated muscle atrophy to some degree [60]. It is important to keep in mind that wrinkles have a multitude of causes, besides muscle contraction, and that treatments of wrinkles based only on the use of BTX-A have poor quality results in the long term [61]. Rohrich (2007) [62] brilliantly demonstrated modern topographic anatomic studies proving the relationship between wrinkles and underlying structures other than muscles, such as arteries, veins, nerves, and septa of fat compartments [62].

The use of BTX-A was first studied by Scott (1973) [63] for the treatment of strabismus by pharmacologic weakening the extraocular muscles [33]. The first described use of the toxin in aesthetic circumstances was by Clark and Berris (1989) [64], but it still carried out the essence of the BoNT-A mechanism of action based on muscle paralysis and atrophy [64]. At some point during the 1990s, Carruthers and Carruthers [65] began to use botulinum toxin type A in full-scale treatments for aesthetic purposes. Since then, the aesthetic focus regarding the use of BoNT-A moved towards removing wrinkles only [65] —a shift in the medical literature on BoTN-A for aesthetics purposes that has persisted until today. We are not underestimating the importance of Carruthers and many other authors that previously studied the use of BoNT-A in aesthetics but, as mentioned above, we intend to provide the aesthetic use of BoNT-A a new perspective. The real mechanism of actions of BoNT-A for aesthetic purposes have been forgotten, to a level where recent publications still focus on the fact that muscle paralysis and muscle atrophy is a complication of the "wrinkle treatment" capacity of BoNT-A instead of its expected effect [66-68].

This systematic review can shed new light on aesthetic BoNT-A treatments basing itself on old, but scientifically correct, concepts of striated muscle contraction physiology, muscle hypertrophy, and muscle atrophy-basic concepts of muscle physiology from reference physiology medical books such as the Guyton and Hall Textbook of Medical Physiology [69].

The results of this systematic review showed evidence that seriated or single BoNT-A muscle injections can cause real atrophy on a short or long-term basis, in animal models and in humans, in skeletal striated muscles of the limbs, facial masticatory muscles, and facial mimetic muscles. Due to only limited good quality data being available, we included animal model studies and human studies, but we know that data extrapolation from animal model studies to humans are, at least, naïve. The sensitivity of animals to BoNT-A has been known for many years to be less than that perceived in humans [70]. There are even differences in sensitivity between rats and mice [71]. On this basis, animal studies must be carefully designed and carefully analyzed, or they cannot be interpreted with respect to human effects [72]. Here we will discuss the results of this systematic review, making clear distinguishment between animal model studies and human studies (Figure 3). 
Thirteen of the sixteen animal studies evaluated muscle quantitative changes and qualitative changes through time after BoNT-A injections

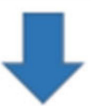

Muscle balance

Structual muscle tissue characteristics through histologic

(optical and electron microscopy) analysis and histochemistry

Muscle imaging

All of them concluded that some degree of muscle atropy occurred, despite the heterogenicity of the studies in relation to animal model used, BoNT-A brand, BoNT-A dose, Muscle group involved and follow-up period.

The partial quantative muscle recovery observed in some animal studies could be attributed not only to muscle regenerationitself, but to muscle substitution for fibrosis and/or fat tissue like demonstrated by Herzog et al. (2007), Fortuna et al. (2011) and Hippokratia et al. (2016)

Fortuna et al. (2015) surprisingly found that the muscle mass was the same across all experimental and conteol group of rabbits

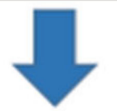

At first glance, a naive view of this article could give the impression of an absent change in muscle balance after 6 months based on total muscle recovery or no muscle loss at all.

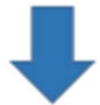


Increasing the number of injections did not produce additional loss in muscle strength and contractile material, as one might have suspected, suggesting that most of the muscle damage effects of BTX-A injection into muscles are caused by the first injection, or that the recovery period between injections was sufficient for partial recovery, thereby offsetting the potential damage induced by each injection.

Genetic alterations related to muscle atrophy/recovery through molecular biology were analyzed by five studies and showed how much impact a single BoNT-A injection can cause on a molecular basis. Mukund (2014) [49] realized that the direct action of BTX-A in skeletal muscle is relatively rapid, inducing dramatic transcriptional adaptation at one week and activating genes in competing pathways of repair and atrophy by gene-related impaired mitochondrial biogenesis.

Much like the findings of animal studies, human studies have also clearly shown atrophy in different muscle types after BTX-A injections. All six human studies that evaluated muscle histology showed atrophy, and when muscle recovery was assessed, there was no full recovery-Borodic (1992) [29] and Schroeder (2009) [41]. Bringing this idea into the context of facial aesthetics, the treatment of the Orbicularis oculi muscle, for example, with BTX-A sporadic injections could atrophy this muscle, but serial and controlled treatments could really maintain a certain degree of atrophy capable of allowing a smile with more open eyes, less caudal traction vector in the cranial part of this muscle postponing gravitational aging, and even give less contribution to the formation of the famous periorbital wrinkles, this time, as a secondary effect. Extrapolations of the powerful tool of muscle atrophy control through time using BTX-A injections could change completely the way BTX-A is used for aesthetic purposes. Dosages, injection intervals, and target muscles would be different from the patterns used nowadays. Instead of planning BTX-A injections to treat wrinkles, a modern anatomy understanding of the facial mimetic muscles as described by Boggio (2017) [74] would be of unparallel importance for aesthetic treatment planning [74]. New approaches for facial aesthetic treatments using BoNT-A could be completely based on mimetic facial muscle interactions and focused on reducing the activity of muscles that enhance gravitational aging (facial depressor muscles), such as the platysma muscle, for example, and preserving antigravitational muscles (elevator facial muscles), such as the frontalis (Figure 4).

After analyzing the results of this paper, we can attempt to answer the questions raised in the introduction (Table 16).

Table 16. Possible and plausible evidence-based answers for the questions raised in the introduction.

\begin{tabular}{ll}
\hline \multicolumn{1}{c}{ Questions } & \multicolumn{1}{c}{ Answers } \\
\hline $\begin{array}{l}\text { Does the muscular impairment for contraction caused by } \\
\text { BoNT-A really treat facial lines or cause muscle atrophy? }\end{array}$ & $\begin{array}{l}\text { Muscle atrophy occurs after BoNT-A injections. Facial } \\
\text { lines are, only in part, treated by BoNT-A injections. }\end{array}$ \\
\hline $\begin{array}{l}\text { What is the relationship betweenf BoNT-A muscle } \\
\text { injections and muscle atrophy in the long term? }\end{array}$ & $\begin{array}{l}\text { Muscles tend to maintain atrophy or have partially } \\
\text { recover after BoNT-A injections. }\end{array}$ \\
\hline $\begin{array}{l}\text { Is it possible to modulate the level of muscle atrophy } \\
\text { through time by using BoNT-A? }\end{array}$ & $\begin{array}{l}\text { At least theoretically it is, and further studies could help } \\
\text { us master this new frontier in facial aesthetics. }\end{array}$ \\
$\begin{array}{l}\text { What if we used muscle atrophy caused by BoNT-A } \\
\text { injections to optimize muscle architecture for facial } \\
\text { aesthetic purposes? }\end{array}$ & $\begin{array}{l}\text { It seems smart to use the atrophy after BoNT-A injections } \\
\text { as a tool for aesthetic purposes instead of the old idea of } \\
\text { an adverse event. }\end{array}$ \\
$\begin{array}{l}\text { What would it be like to reinterpret articles written in the } \\
\text { last 30 years that focused mainly on facial lines unveiling } \\
\text { this concept of muscle atrophy? How many less } \\
\text { subjective opportunities would arise? How would classic } \\
\text { BoNT-A injections techniques would be impacted? }\end{array}$ & $\begin{array}{l}\text { We are sure that understanding BoNT-A as a muscle } \\
\text { atrophy tool for aesthetic purposes will bring us to new } \\
\text { treatments. }\end{array}$ \\
\hline
\end{tabular}




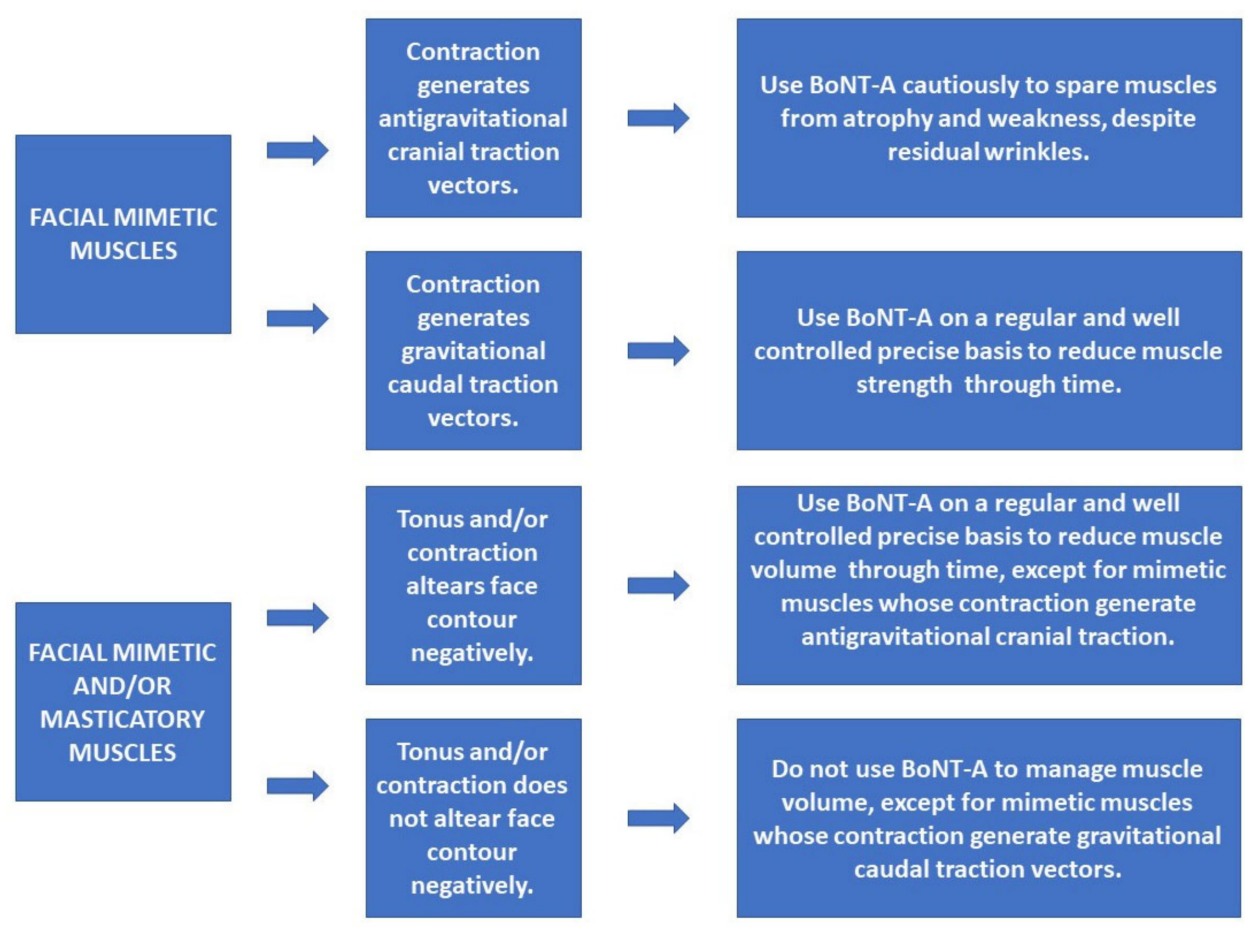

Figure 4. New approaches for facial aesthetic treatments using BoNT-A. The human imaging studies, similar to the animal studies, also show muscle atrophy and volume reduction. Koerte (2013) [47] showed a sustained atrophy and volume loss of approximately $50 \%$ in the procerus muscle. New perspectives on aesthetics BoNT-A treatments should consider not only facial mimetic muscles and their strength in relation to gravitational or antigravitational contraction vectors, but also their volume. Muscle volume control is also of aesthetic importance. The understanding that some degree of muscle volume reduction would bring positive aesthetic aspects for some mimetic muscles, such as the procerus and corrugators and some masticatory muscles such as the masseter, would also change the current BoNT-A injections patterns. On the other hand, some muscles should be spared from volume loss, such as the frontalis and the lateral aspect of the orbicularis oculi, to avoid facial skeletonization.

\section{Conclusions}

This systematic review showed evidence that seriated or single BoNT-A muscle injections can cause real muscle atrophy on a short or long-term basis, in animal models and in humans, in skeletal striated muscles of the limbs, facial masticatory muscles, and facial mimetic muscles. Theoretically, muscular architecture reprogramming is a possible new approach in aesthetics. Depressor facial muscles could be targeted to have some degree of atrophy with BoNT-A injections, while elevator facial muscles could be spared to some degree to maintain antigravitational traction forces and facilitate a lift effect.

Author Contributions: Conceptualization, A.D.N.; methodology, A.D.N.; validation, A.D.N.; formal analysis, A.D.N.; investigation, A.D.N.; data curation, A.D.N.; writing-original draft preparation, A.D.N.; writing-review and editing, R.F.B., S.E. and G.E.L.F.; All authors have read and agreed to the published version of the manuscript.

Funding: This research received no external funding.

Conflicts of Interest: The authors declare no conflict of interest. 


\section{References}

1. Gart, M.S.; Gutowski, K.A. Overview of Botulinum Toxins for Aesthetic Uses. Clin. Plast. Surg. 2016, 43, 459-471. [CrossRef]

2. Cavallini, M.; Cirillo, P.; Fundarò, S.P.; Quartucci, S.; Sciuto, C.; Sito, G.; Tonini, D.; Trocchi, G.; Signorini, M. Safety of botulinum toxin A in aesthetic treatments: A systematic review of clinical studies. Dermatol. Surg. 2014, 40, 525-536. [CrossRef]

3. Ministério da Saúde Consultoria Jurídica/Advocacia Geral da União 1 Nota Técnica N ${ }^{\circ} N^{\prime}$ ota Técnica N³42/2014. Available online: https://portalarquivos2.saude.gov.br/images/pdf/2014/setembro/17/Toxina-botul--nica-tipo-A.pdf (accessed on 28 July 2019).

4. Monheit, G. Neurotoxins: Current Concepts in Cosmetic Use on the Face and Neck-Upper Face (Glabella, Forehead, and Crow's Feet). Plast. Reconstr. Surg. 2015, 136, 72S-75S. [CrossRef] [PubMed]

5. Bonaparte, J.P.; Ellis, D.; Quinn, J.G.; Rabski, J.; Hutton, B. A Comparative Assessment of Three Formulations of Botulinum Toxin Type A for Facial Rhytides: A Systematic Review with Meta-Analyses. Plast. Reconstr. Surg. 2016, 137, 1125-1140. [CrossRef]

6. Brin, M.F.; James, C.; Maltman, J. Botulinum toxin type A products are not interchangeable: A review of the evidence. Biologics 2014, 8, 227-241. [CrossRef] [PubMed]

7. Albanese, A. Terminology for preparations of botulinum neurotoxins: What a difference a name makes. JAMA 2011, 305, 89-90, Erratum in JAMA 2011, 305, 1544. [CrossRef] [PubMed]

8. Wilson, A.J.; Chang, B.; Taglienti, A.J.; Chin, B.C.; Chang, C.S.; Folsom, N.; Percec, I. A Quantitative Analysis of OnabotulinumtoxinA, AbobotulinumtoxinA, and IncobotulinumtoxinA: A Randomized, Double-Blind, Prospective Clinical Trial of Comparative Dynamic Strain Reduction. Plast. Reconstr. Surg. 2016, 137, 1424-1433. [CrossRef]

9. Burgen, A.S.; Dickens, F.; Zatman, L.J. The action of botulinum toxin on the neuro-muscular junction. J. Physiol. 1949, 109, 10-24. [CrossRef] [PubMed]

10. Simpson, L.L. The origin, structure, and pharmacological activity of botulinum toxin. Pharmacol. Rev. 1981, 33, 155-188.

11. Aoki, K.R. Pharmacology and immunology of botulinum toxin type A. Clin. Dermatol. 2003, 21, 476-480. [CrossRef]

12. Pellizzari, R.; Rossetto, O.; Schiavo, G.; Montecucco, C. Tetanus and botulinum neurotoxins: Mechanism of action and therapeutic uses. Philos. Trans. R. Soc. Lond. Ser. B Biol. Sci. 1999, 354, 259-268. [CrossRef]

13. Schiavo, G.; Matteoli, M.; Montecucco, C. Neurotoxins affecting neuroexocytosis. Physiol. Rev. 2000, 80, 717-766. [CrossRef]

14. Lacy, D.B.; Tepp, W.; Cohen, A.C.; DasGupta, B.R.; Stevens, R.C. Crystal structure of botulinum neurotoxin type A and implications for toxicity. Nat. Struct. Biol. 1998, 5, 898-902. [CrossRef]

15. Koriazova, L.K.; Montal, M. Translocation of botulinum neurotoxin light chain protease through the heavy chain channel. Nat. Struct. Biol. 2003, 10, 13-18. [CrossRef] [PubMed]

16. Rizo, J.; Südhof, T.C. Snares and Munc18 in synaptic vesicle fusion. Nat. Rev. Neurosci. 2002, 3, 641-653. [CrossRef]

17. Pingel, J.; Nielsen, M.S.; Lauridsen, T.; Rix, K.; Bech, M.; Alkjaer, T.; Andersen, I.T.; Nielsen, J.B.; Feidenhansl, R. Injection of high dose botulinum-toxin A leads to impaired skeletal muscle function and damage of the fibrilar and non-fibrilar structures. Sci. Rep. 2017, 7, 14746. [CrossRef] [PubMed]

18. Nigam, P.K.; Nigam, A. Botulinum toxin. Indian J. Dermatol. 2010, 55, 8-14. [CrossRef] [PubMed]

19. De Paiva, A.; Meunier, F.A.; Molgó, J.; Aoki, K.R.; Dolly, J.O. Functional repair of motor endplates after botulinum neurotoxin type A poisoning: Biphasic switch of synaptic activity between nerve sprouts and their parent terminals. Proc. Natl. Acad. Sci. USA 1999, 96, 3200-3205. [CrossRef]

20. Holland, R.L.; Brown, M.C. Nerve growth in botulinum toxin poisoned muscles. Neuroscience 1981, 6, 1167-1179. [CrossRef]

21. Hopkins, W.G.; Brown, M.C. The distribution of nodal sprouts in a paralysed or partly denervated mouse muscle. Neuroscience 1982, 7, 37-44. [CrossRef]

22. Pamphlett, R. Early terminal and nodal sprouting of motor axons after botulinum toxin. J. Neurol. Sci. 1989, 92, 181-192. [CrossRef]

23. Anderson, D.M.; Kumar, V.R.; Arper, D.L.; Kruger, E.; Bilir, S.P.; Richardson, J.S. Cost savings associated with timely treatment of botulism with botulism antitoxin heptavalent product. PLOS ONE 2019, 14, e0224700. [CrossRef] [PubMed]

24. Arnon, S.S.; Schechter, R.; Inglesby, T.V.; Henderson, D.A.; Bartlett, J.G.; Ascher, M.S.; Eitzen, E.; Fine, A.D.; Hauer, J.; Layton, M.; et al. Working Group on Civilian Biodefense. Botulinum toxin as a biological weapon: Medical and public health management. JAMA 2001, 285, 1059-1070, Erratum in JAMA 2001, 285, 2081. [CrossRef]

25. Chalk, C.H.; Benstead, T.J.; Pound, J.D.; Keezer, M.R. Medical treatment for botulism. Cochrane Database Syst. Rev. 2019, 4, CD008123. [CrossRef]

26. Lin, L.; Olson, M.E.; Eubanks, L.M.; Janda, K.D. Strategies to Counteract Botulinum Neurotoxin A: Nature's Deadliest Biomolecule. Acc. Chem. Res. 2019, 52, 2322-2331. [CrossRef]

27. Moher, D.; Liberati, A.; Tetzlaff, J.; Altman, D.G.; PRISMA Group. Preferred reporting items for systematic reviews and meta-analyses: The PRISMA statement. Version 2. BMJ 2009, 339, b2535. [CrossRef]

28. Vandenbroucke, J.P.; von Elm, E.; Altman, D.G.; Gøtzsche, P.C.; Mulrow, C.D.; Pocock, S.J.; Poole, C.; Schlesselman, J.J.; Egger, M. STROBE Initiative. Strengthening the Reporting of Observational Studies in Epidemiology (STROBE): Explanation and elaboration. Int. J. Surg. 2014, 12, 1500-1524. [CrossRef]

29. Borodic, G.E.; Ferrante, R. Effects of repeated botulinum toxin injections on orbicularis oculi muscle. J Clin Neuroophthalmol. 1992, 12, 121-127. [CrossRef] 
30. Hamjian, J.A.; Walker, F.O. Serial neurophysiological studies of intramuscular botulinum-A toxin in humans. Muscle Nerve. 1994, 17, 1385-1392. [CrossRef] [PubMed]

31. Ansved, T.; Odergren, T.; Borg, K. Muscle fiber atrophy in leg muscles after botulinum toxin type A treatment of cervical dystonia. Neurology 1997, 48, 1440-1442. [CrossRef]

32. Fanucci, E.; Masala, S.; Sodani, G.; Varrucciu, V.; Romagnoli, A.; Squillaci, E.; Simonetti, G. CT-guided injection of botulinic toxin for percutaneous therapy of piriformis muscle syndrome with preliminary MRI results about denervative process. Eur. Radiol. 2001, 11, 2543-2548. [CrossRef]

33. To, E.W.; Ahuja, A.T.; Ho, W.S.; King, W.W.; Wong, W.K.; Pang, P.C.; Hui, A.C. A prospective study of the effect of botulinum toxin A on masseteric muscle hypertrophy with ultrasonographic and electromyographic measurement. Br. J. Plast Surg. 2001, 54, 197-200. [CrossRef]

34. Kim, N.H.; Chung, J.H.; Park, R.H.; Park, J.B. The use of botulinum toxin type A in aesthetic mandibular contouring. Plast. Reconstr. Surg. 2005, 115, 919-930. [CrossRef]

35. Shen, J.; Ma, J.; Lee, C.; Smith, B.P.; Smith, T.L.; Tan, K.H.; Koman, L.A. How muscles recover from paresis and atrophy after intramuscular injection of botulinum toxin A: Study in juvenile rats. J. Orthop. Res. 2006, 24, 1128-1135. [CrossRef]

36. Singer, B.J.; Silbert, P.L.; Dunne, J.W.; Song, S.; Singer, K.P. An open label pilot investigation of the efficacy of Botulinum toxin type A [Dysport] injection in the rehabilitation of chronic anterior knee pain. Disabil. Rehabil. 2006, 28, 707-713. [CrossRef]

37. Herzog, W.; Longino, D. The role of muscles in joint degeneration and osteoarthritis. J. Biomech. 2007, 40 (Suppl. 1), S54-S63, Erratum in J. Biomech. 2008, 41, 2332-2335. [CrossRef] [PubMed]

38. Frick, C.G.; Richtsfeld, M.; Sahani, N.D.; Kaneki, M.; Blobner, M.; Martyn, J.A. Long-term effects of botulinum toxin on neuromuscular function. Anesthesiology 2007, 106, 1139-1146. [CrossRef] [PubMed]

39. Kwon, T.G.; Park, H.S.; Lee, S.H.; Park, I.S.; An, C.H. Influence of unilateral masseter muscle atrophy on craniofacial morphology in growing rabbits. J. Oral Maxillofac. Surg. 2007, 65, 1530-1537. [CrossRef]

40. Lee, C.J.; Kim, S.G.; Kim, Y.J.; Han, J.Y.; Choi, S.H.; Lee, S.I. Electrophysiologic change and facial contour following botulinum toxin A injection in square faces. Plast Reconstr. Surg. 2007, 120, 769-778. [CrossRef] [PubMed]

41. Schroeder, A.S.; Ertl-Wagner, B.; Britsch, S.; Schröder, J.M.; Nikolin, S.; Weis, J.; Müller-Felber, W.; Koerte, I.; Stehr, M.; Berweck, S.; et al. Muscle biopsy substantiates long-term MRI alterations one year after a single dose of botulinum toxin injected into the lateral gastrocnemius muscle of healthy volunteers. Mov. Disord. 2009, 24, 1494-1503. [CrossRef]

42. Babuccu, B.; Babuccu, O.; Yurdakan, G.; Ankarali, H. The effect of the Botulinum toxin-A on craniofacial development: An experimental study. Ann. Plast Surg. 2009, 63, 449-456. [CrossRef]

43. Tsai, F.C.; Hsieh, M.S.; Chou, C.M. Comparison between neurectomy and botulinum toxin A injection for denervated skeletal muscle. J. Neurotrauma. 2010, 27, 1509-1516. [CrossRef] [PubMed]

44. Fortuna, R.; Vaz, M.A.; Youssef, A.R.; Longino, D.; Herzog, W. Changes in contractile properties of muscles receiving repeat injections of botulinum toxin (Botox). J. Biomech. 2011, 44, 39-44. [CrossRef] [PubMed]

45. Fortuna, R.; Horisberger, M.; Vaz, M.A.; Van der Marel, R.; Herzog, W. The effects of electrical stimulation exercise on muscles injected with botulinum toxin type-A (botox). J. Biomech. 2013, 46, 36-42. [CrossRef]

46. Van Campenhout, A.; Verhaegen, A.; Pans, S.; Molenaers, G. Botulinum toxin type A injections in the psoas muscle of children with cerebral palsy: Muscle atrophy after motor end plate-targeted injections. Res. Dev. Disabil. 2013, 34, 1052-1058. [CrossRef] [PubMed]

47. Koerte, I.K.; Schroeder, A.S.; Fietzek, U.M.; Borggraefe, I.; Kerscher, M.; Berweck, S.; Reiser, M.; Ertl-Wagner, B.; Heinen, F. Muscle atrophy beyond the clinical effect after a single dose of OnabotulinumtoxinA injected in the procerus muscle: A study with magnetic resonance imaging. Dermatol. Surg. 2013, 39, 761-765. [CrossRef]

48. Fortuna, R.; Horisberger, M.; Vaz, M.A.; Herzog, W. Do skeletal muscle properties recover following repeat onabotulinum toxin A injections? J. Biomech. 2013, 46, 2426-2433. [CrossRef]

49. Mukund, K.; Mathewson, M.; Minamoto, V.; Ward, S.R.; Subramaniam, S.; Lieber, R.L. Systems analysis of transcriptional data provides insights into muscle's biological response to botulinum toxin. Muscle Nerve. 2014, 50, 744-758. [CrossRef] [PubMed]

50. Fortuna, R.; Vaz, M.A.; Sawatsky, A.; Hart, D.A.; Herzog, W. A clinically relevant BTX-A injection protocol leads to persistent weakness, contractile material loss, and an altered mRNA expression phenotype in rabbit quadriceps muscles. J. Biomech. 2015, 48, 1700-1706. [CrossRef] [PubMed]

51. Caron, G.; Marqueste, T.; Decherchi, P. Long-Term Effects of Botulinum Toxin Complex Type A Injection on Mechano- and Metabo-Sensitive Afferent Fibers Originating from Gastrocnemius Muscle. PLoS ONE. 2015, 10, e0140439. [CrossRef]

52. Valentine, J.; Stannage, K.; Fabian, V.; Ellis, K.; Reid, S.; Pitcher, C.; Elliott, C. Muscle histopathology in children with spastic cerebral palsy receiving botulinum toxin type A. Muscle Nerve 2016, 53, 407-414. [CrossRef]

53. Li, J.; Allende, A.; Martin, F.; Fraser, C.L. Histopathological changes of fibrosis in human extra-ocular muscle caused by botulinum toxin A. J. AAPOS 2016, 20, 544-546. [CrossRef]

54. Kocaelli, H.; Yaltirik, M.; Ayhan, M.; Aktar, F.; Atalay, B.; Yalcin, S. Ultrastructural evaluation of intramuscular applied botulinum toxin type A in striated muscles of rats. Hippokratia 2016, 20, 292-298. [PubMed]

55. Hart, D.A.; Fortuna, R.; Herzog, W. Messenger RNA profiling of rabbit quadriceps femoris after repeat injections of botulinum toxin: Evidence for a dynamic pattern without further structural alterations. Muscle Nerve 2018, 57, 487-493. [CrossRef] [PubMed] 
56. Han, S.K.; Lee, Y.; Hong, J.J.; Yeo, H.G.; Seo, J.; Jeon, C.Y.; Jeong, K.J.; Jin, Y.B.; Kang, P.; Lee, S.; et al. In vivo study of paraspinal muscle weakness using botulinum toxin in one primate model. Clin. Biomech. 2018, 53, 1-6. [CrossRef]

57. Alexander, C.; Elliott, C.; Valentine, J.; Stannage, K.; Bear, N.; Donnelly, C.J.; Shipman, P.; Reid, S. Muscle volume alterations after first botulinum neurotoxin A treatment in children with cerebral palsy: A 6-month prospective cohort study. Dev. Med. Child. Neurol. 2018, 60, 1165-1171. [CrossRef]

58. Lima, W.; Salles, A.G.; Faria, J.C.M.; Nepomuceno, A.C.; Salomone, R.; Krunn, P.; Gemperli, R. Contralateral Botulinum Toxin Improved Functional Recovery after Tibial Nerve Repair in Rats. Plast. Reconstr. Surg. 2018, 142, 1511-1519. [CrossRef] [PubMed]

59. International Society of Aesthetic Plastic Surgery. The International Study on Aesthetic/Cosmetic Procedures Performed in 2018. International Society of Aesthetic Plastic Surgery. 2018. Available online: https://www.isaps.org/wp-content/uploads/2019/12/ ISAPS-Global-Survey-Results-2018-new.pdf (accessed on 10 July 2020).

60. Hastings-Ison, T.; Graham, H.K. Atrophy and hypertrophy following injections of botulinum toxin in children with cerebral palsy. Dev. Med. Child. Neurol. 2013, 55, 778-779. [CrossRef]

61. Manríquez, J.J.; Cataldo, K.; Vera-Kellet, C.; Harz-Fresno, I. Wrinkles. BMJ Clin. Evid. 2014, $2014,1711$.

62. Rohrich, R.J.; Pessa, J.E. The fat compartments of the face: Anatomy and clinical implications for cosmetic surgery. Plast. Reconstr. Surg. 2007, 119, 2219-2227, discussion 2228-2231. [CrossRef]

63. Scott, A.B.; Rosenbaum, A.; Collins, C.C. Pharmacologic weakening of extraocular muscles. Invest. Ophthalmol. 1973, 12, 924-927. [PubMed]

64. Clark, R.P.; Berris, C.E. Botulinum toxin: A treatment for facial asymmetry caused by facial nerve paralysis. Plast. Reconstr. Surg. 1989, 84, 353-355. [CrossRef]

65. Carruthers, A.; Carruthers, J. History of the cosmetic use of Botulinum A exotoxin. Dermatol. Surg. 1998, 24, 1168-1170. [CrossRef] [PubMed]

66. Salari, M.; Sharma, S.; Jog, M.S. Botulinum Toxin Induced Atrophy: An Uncharted Territory. Toxins 2018, 10, 313. [CrossRef] [PubMed]

67. Yiannakopoulou, E. Serious and long-term adverse events associated with the therapeutic and cosmetic use of botulinum toxin. Pharmacology 2015, 95, 65-69. [CrossRef]

68. Durand, P.D.; Couto, R.A.; Isakov, R.; Yoo, D.B.; Azizzadeh, B.; Guyuron, B.; Zins, J.E. Botulinum Toxin and Muscle Atrophy: A Wanted or Unwanted Effect. Aesthet. Surg. J. 2016, 36, 482-487. [CrossRef]

69. Khonsary, S.A. Guyton and Hall: Textbook of Medical Physiology. Surg. Neurol. Int. 2017, 8, 275. [CrossRef]

70. Pirazzini, M.; Rossetto, O.; Eleopra, R.; Montecucco, C. Botulinum Neurotoxins: Biology, Pharmacology, and Toxicology. Pharmacol. Rev. 2017, 69, 200-235. [CrossRef] [PubMed]

71. Rosales, R.L.; Bigalke, H.; Dressler, D. Pharmacology of botulinum toxin: Differences between type A preparations. Eur. J. Neurol. 2006, 13, 2-10. [CrossRef]

72. Pickett, A. Animal studies with botulinum toxins may produce misleading results. Anesth. Analg. 2012, 115, 736-737. [CrossRef]

73. Damiano, D.L.; Moreau, N. Muscle thickness reflects activity in CP but how well does it represent strength? Dev. Med. Child Neurol. 2008, 50, 88. [CrossRef] [PubMed]

74. Boggio, R.F. Dynamic Model of Applied Facial Anatomy with Emphasis on Teaching of Botulinum Toxin A. Plast. Reconstr. Surg. Glob. Open 2017, 5, e1525. [CrossRef] [PubMed] 